\title{
Dysregulated mTORC1 renders cells critically dependent on desaturated lipids for survival under tumor-like stress
}

\author{
Regina M. Young, ${ }^{1,2}$ Daniel Ackerman, ${ }^{1}$ Zachary L. Quinn, ${ }^{1,3}$ Anthony Mancuso ${ }^{4}$ Michaela Gruber, ${ }^{1,5}$ \\ Liping Liu, ${ }^{1,3,6}$ Dionysios N. Giannoukos, ${ }^{1,3}$ Ekaterina Bobrovnikova-Marjon, ${ }^{1,7}$ J. Alan Diehl, ${ }^{1,2}$ \\ Brian Keith, $^{1,2}$ and M. Celeste Simon ${ }^{1,3,8}$ \\ ${ }^{1}$ Abramson Family Cancer Research Institute, ${ }^{2}$ Department of Cancer Biology, ${ }^{3}$ Howard Hughes Medical Institute, ${ }^{4}$ Department \\ of Radiology, Perelman School of Medicine, University of Pennsylvania, Philadelphia, Pennsylvania 19104, USA
}

\begin{abstract}
Solid tumors exhibit heterogeneous microenvironments, often characterized by limiting concentrations of oxygen $\left(\mathrm{O}_{2}\right)$, glucose, and other nutrients. How oncogenic mutations alter stress response pathways, metabolism, and cell survival in the face of these challenges is incompletely understood. Here we report that constitutive mammalian target of rapamycin complex 1 (mTORC1) activity renders hypoxic cells dependent on exogenous desaturated lipids, as levels of de novo synthesized unsaturated fatty acids are reduced under low $\mathrm{O}_{2}$. Specifically, we demonstrate that hypoxic Tsc $2^{--}$(tuberous sclerosis complex $2^{--}$) cells deprived of serum lipids exhibit a magnified unfolded protein response (UPR) but fail to appropriately expand their endoplasmic reticulum (ER), leading to inositol-requiring protein-1 (IRE1)-dependent cell death that can be reversed by the addition of unsaturated lipids. UPR activation and apoptosis were also detected in Tsc2-deficient kidney tumors. Importantly, we observed this phenotype in multiple human cancer cell lines and suggest that cells committed to unregulated growth within ischemic tumor microenvironments are unable to balance lipid and protein synthesis due to a critical limitation in desaturated lipids.
\end{abstract}

[Keywords: mTORC1; ER stress; UPR; hypoxia; lipid desaturation; tumor microenvironment]

Supplemental material is available for this article.

Received June 15, 2012; revised version accepted April 23, 2013.

Normal mammalian cells engage multiple signaling pathways to regulate their metabolism in response to changing levels of available glucose, amino acids, and oxygen $\left(\mathrm{O}_{2}\right)$. In contrast, most cancer cells display dysregulated metabolic activity as a consequence of oncogene activation and tumor suppressor loss (Menon and Manning 2008). We hypothesized that genetic alterations that drive cell growth and proliferation could commit tumor cells to a metabolic program that proves unsupportable under the variable conditions of nutrient, growth factor, and $\mathrm{O}_{2}$ deprivation characteristic of poorly vascularized solid tumors. To test this hypothesis, we investigated macromolecular biosynthesis, stress response signaling, and survival in cells with constitutive activation of the mammalian target of rapamycin complex 1 (mTORC1)

Present addresses: ${ }^{5}$ Roche Austria GmbH, Engelhorngasse 3, 1210 Vienna, Austria; ${ }^{6}$ Merck and Co., Inc., 770 Sumneytown Pike, West Point, PA 19486, USA; ${ }^{7}$ Agios Pharmaceuticals, 38 Sidney Street, Cambridge, MA, 02139 USA

${ }^{8}$ Corresponding author

E-mail celeste2@mail.med.upenn.edu

Article is online at http://www.genesdev.org/cgi/doi/10.1101/gad.198630.112. upon challenge with a combination of tumor-associated microenvironmental stresses.

mTORC1 is a multiprotein serine/threonine kinase that promotes cell growth by regulating multiple biosynthetic processes, including ribosome biogenesis and protein and lipid synthesis (Ozcan et al. 2008; Duvel et al. 2010; Egan et al. 2011; Kim and Guan 2011). Importantly, mTORC1 activity is exquisitely sensitive to changes in extracellular nutrient levels and intracellular bioenergetics (Wullschleger et al. 2006), thereby modulating ATPintensive biosynthetic processes to match available substrate concentrations. The tuberous sclerosis complex proteins 1 and $2(\mathrm{TSCl} / 2)$ are the primary link between extracellular signals and mTORC1 activity. Loss-of-function mutations in either TSC1 or TSC2 result in constitutive mTORC1 activation and are the genetic cause of the human TSC hamartomatous syndrome (Crino et al. 2006). Interestingly, the loss of other tumor suppressors (LKB1 and NF1) or the activation of frequently mutated oncogenes (AKT, PI3K, and Ras) also lead to aberrantly elevated mTORC1 activity (Laplante and Sabatini 2012), which in turn contributes to the unregulated growth of transformed cells (Duvel et al. 2010). 
Regulation of mTORC1 activity by TSC proteins is mediated by distinct phosphorylation events in response to complex cellular signals. For example, growth factor and cytokine signaling stimulate AKT, which phosphorylates and represses TSC2, thereby activating mTORC1 and promoting cell growth (Manning et al. 2002). Conversely, under conditions of low intracellular energy charge, AMP-activated kinase (AMPK)-mediated phosphorylation of TSC2 augments MTORC1 inhibition, thus preserving ATP levels by reducing mTORC1-dependent protein synthesis and protecting cells from energy deprivation-induced apoptosis (Inoki et al. 2003). Consequently, TSC2-deficient cells fail to attenuate mTORC1-dependent anabolic processes and display elevated rates of apoptosis when challenged with glucose deprivation (Choo et al. 2010), demonstrating the critical importance of suppressing mTORC1 activity in the face of ATP depletion.

mTORC1 activity also influences a variety of critical cellular stress responses. For example, mTORC1 and hypoxia-inducible factor (HIF) collaborate to respond to cellular $\mathrm{O}_{2}$ deprivation (hypoxia). Dysregulated mTORC1 activity promotes translation of HIF- $1 \alpha$ mRNA (Duvel et al. 2010), thereby enhancing HIF-1 $\alpha$-dependent increases in glucose transport and glycolysis (Seagroves et al. 2001) at the expense of oxidative phosphorylation. Although mTORC1 activity promotes HIF- $1 \alpha$-dependent glycolysis, HIF- $1 \alpha$ can in turn attenuate mTORC1 activity by inducing the expression of REDD1 (Brugarolas et al. 2004), which releases sequestered TSC2 from the scaffold protein 14-3-3 (DeYoung et al. 2008), suppressing ATPintensive mTORC1-dependent macromolecular biosynthesis under severely hypoxic conditions. It is also important to note that mTORC1 signaling is regulated by additional TSC-independent mechanisms (Liu et al. 2006; Gwinn et al. 2008).

Severely hypoxic cells experience endoplasmic reticulum (ER) stress, partly because $\mathrm{O}_{2}$ is required for oligosaccharide modifications and disulfide bond formation (Wouters and Koritzinsky 2008). Constitutive mTORC1 activation also induces ER stress by promoting unregulated protein accumulation (Ozcan et al. 2008). Both hypoxia and mTORC1 dysregulation therefore engage the unfolded protein response (UPR) and consequent autophosphorylation of its downstream sensors (protein kinase RNA-like ER kinase [PERK] and inositol-requiring protein-1 $\alpha[\operatorname{IRE} 1 \alpha])$, which function to reduce protein load and enhance protein-folding capacity. If unresolved, persistent UPR activation can ultimately trigger apoptosis downstream from PERK and IRE $1 \alpha$ engagement. These and other data indicate that complex and dynamic interactions between mTORC1 activity and cellular stress response pathways play a critical role in modulating biosynthesis to maintain cellular homeostasis and survival under conditions of limiting $\mathrm{O}_{2}$ and nutrients.

Growth factor, $\mathrm{O}_{2}$, and glucose levels vary greatly within subdomains of solid tumors and across a broad spectrum of tumor classes and grades (Bertout et al. 2008). Given the frequency of aberrant mTORC1 activation in cancer cells, we hypothesized that these complex microenvironmental conditions would alter mTORC1-dependent stress response signaling, macromolecular biosynthesis, and cell survival. We report here that exposing Tsc2-null cells to complex tumor-like stresses produced an exacerbated UPR, resulting in mTORC1- and IRE1 $\alpha$-dependent cell death. Importantly, similar responses were observed in spontaneous TSC2-deficient kidney tumors arising in heterozygous $\mathrm{TsC}^{+/-}$mice. We demonstrate that under specific conditions of serum and $\mathrm{O}_{2}$ limitation, Tsc2-null mouse embryonic fibroblasts (MEFs) die from a lack of unsaturated fatty acids, which are required for ER expansion to accommodate elevated levels of unfolded proteins. These data reveal a surprising and novel connection between lipid depletion and the UPR and demonstrate that mTORC1-driven cells are dependent on serum lipids for survival under low $\mathrm{O}_{2}$. Intriguingly, multiple cancer cell lines derived from distinct tissue types are also dependent on exogenously supplied unsaturated fatty acids for survival when challenged with $\mathrm{O}_{2}$ and serum deprivation.

\section{Results}

Constitutive mTORC1 activity promotes cell death under tumor-like stress

We used colony-forming assays to investigate the effects of constitutive mTORC1 activity on cell survival under variable conditions of tumor-like ischemic stress. A null allele of $p 53$ was incorporated into $\mathrm{Tsc}^{+/+}$and $\mathrm{Tsc}^{-/-}$ genetic backgrounds, as p53 is frequently mutated in human cancers (Vousden and Prives 2009) and primary $\mathrm{Tsc}^{-/-}$MEFs rapidly senesce in culture (Zhang et al. 2003). $\mathrm{Tsc}^{+/+}, \mathrm{p} 53^{-/-}$and $\mathrm{Tsc} 2^{-/-}, \mathrm{p} 53^{-/-}$MEFs were exposed to combinations of serum, $\mathrm{O}_{2}$, and glucose limitation for $48 \mathrm{~h}$ and then grown in complete medium for $7 \mathrm{~d}$. $T s c 2^{+/+}, p 53^{-/-}$ MEFs survived limitation of serum alone, $\mathrm{O}_{2}$ alone $13 \%$, $1.5 \%$, and $0.5 \%)$, and the combination of serum and glucose limitation (Fig. 1A). In contrast, $T s c 2^{-/-}, p 53^{-/-}$MEFs succumbed to conditions of combined serum and $\mathrm{O}_{2}$ depletion $(0.5 \%)$ irrespective of glucose limitation (Fig. 1A). To further characterize this apparent mTOR-dependent loss of cell viability, Tsc2 ${ }^{+/+}, p 53^{-/-}$and Tsc2 ${ }^{-/-}, p 53^{-/-}$MEFs were exposed to stress conditions for $48 \mathrm{~h}$, and viability was assessed directly by flow cytometry (Supplemental Fig. S1A,B). Under either "SO" conditions $(0.5 \%$ serum and $\left.0.5 \% \mathrm{O}_{2}\right)$ or "SOG" conditions $(0.5 \%$ serum, $0.5 \%$ $\mathrm{O}_{2}$, and $0.5 \mathrm{mM}$ glucose), Tsc $2^{+/+}, \mathrm{p} 53^{-/-}$MEFs exhibited significantly enhanced viability $(89.2 \%$ and $66.8 \%)$ compared with $T s c 2^{-/-}$, $p 53^{-/-}$MEFs $(42.3 \%$ and $46.8 \%$ ) (Fig. 1B); therefore, in subsequent experiments, we focused on these specific stress conditions (SO and SOG) to elucidate the role of $\mathrm{mTOR}$ in ischemic cell death.

The mTORC1-specific inhibitor rapamycin (Yip et al. 2010) as well as combined mTORC1/mTORC2 inhibitor torin (Guertin and Sabatini 2007; Thoreen et al. 2009) rescued the survival of $T s c 2^{-/-}, p 53^{-/-}$MEFs after $48 \mathrm{~h}$ of exposure to either SO or SOG conditions (Fig. 1C; Supplemental Fig. S1C), suggesting that constitutive mTORC1 activation is responsible for promoting cell death under ischemic stress. To confirm that loss of TSC2 impacts viability under tumor-like stress, we analyzed 

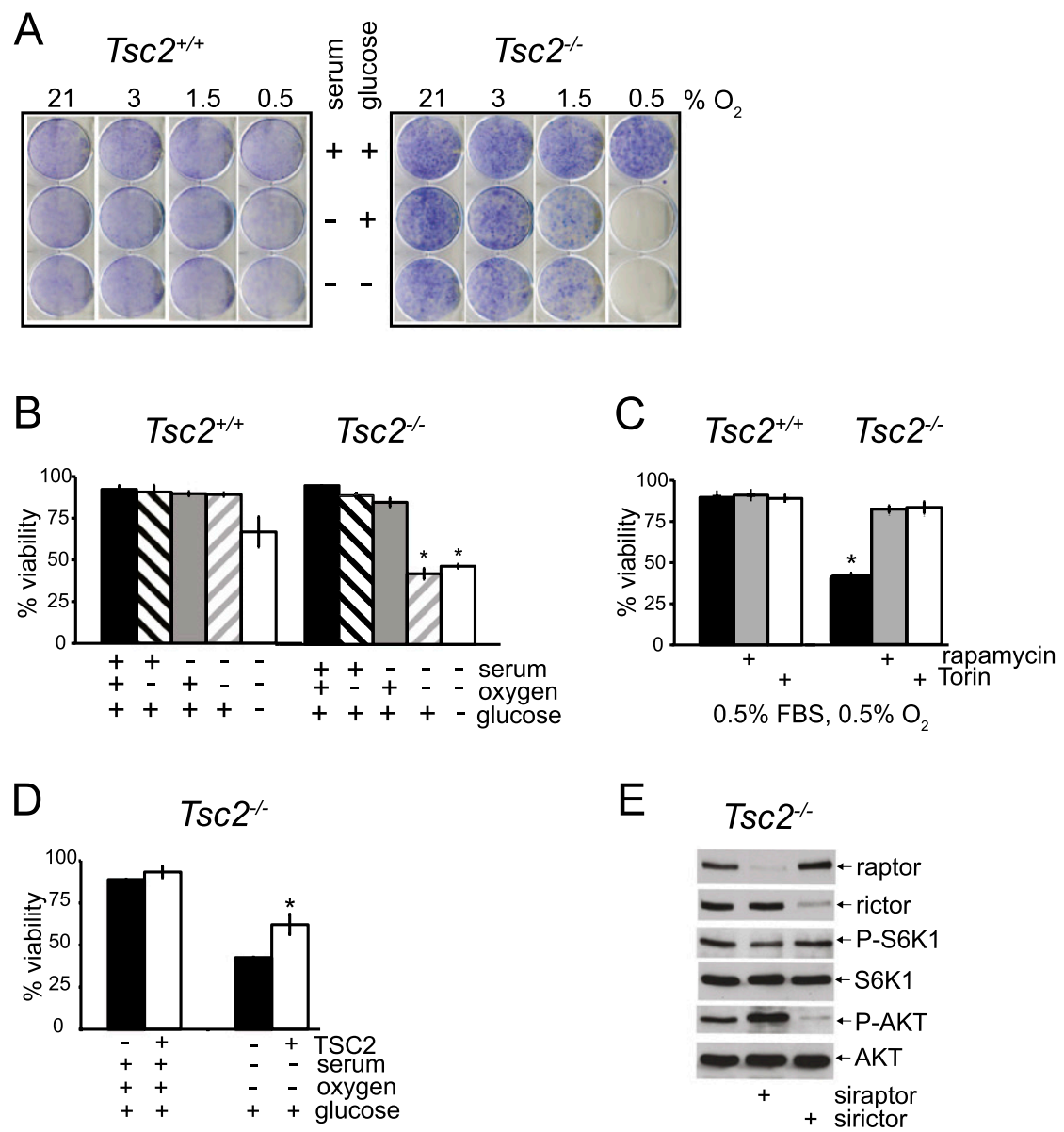

$0.5 \% \mathrm{FBS}, 0.5 \% \mathrm{O}_{2}$
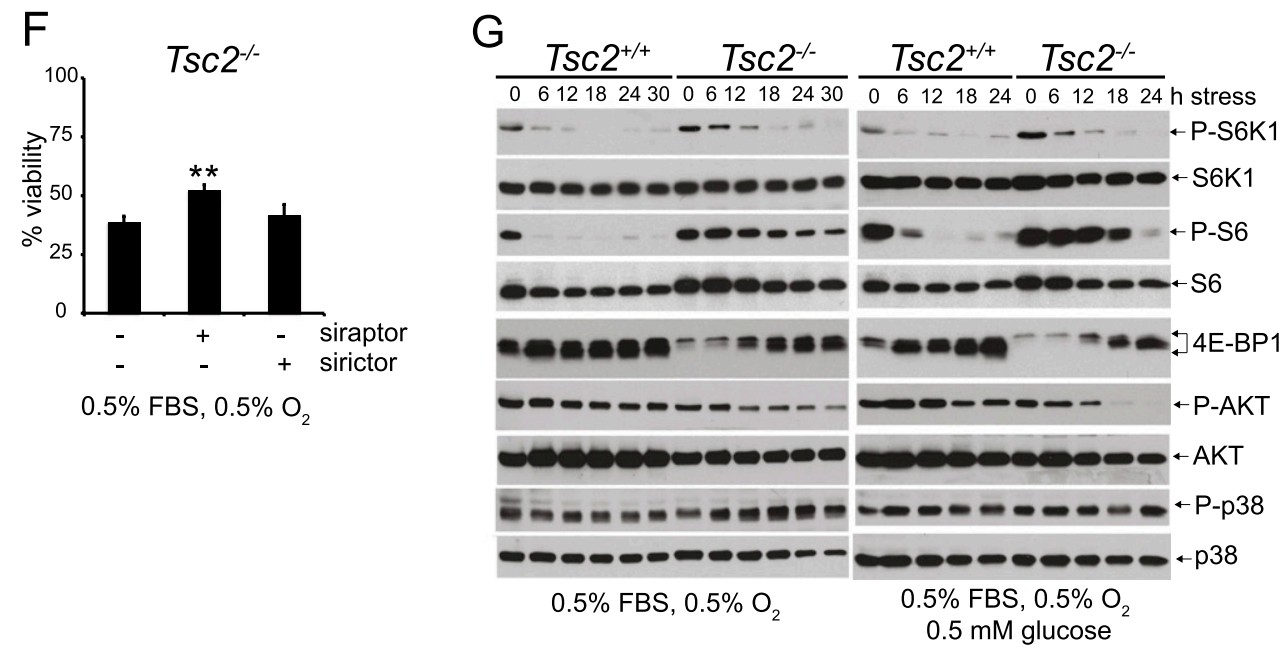

Figure 1. Constitutive mTOR activity promotes cell death under tumor-like stress. $(A)$ To assay the survival of $T s c 2^{+/+}, p 53^{-/-}$and $\mathrm{Tsc2}^{-/-}$, $\mathrm{p} 53^{-/-}$MEFs under stress, cells were exposed to $21 \%, 3 \%, 1.5 \%$, or $0.5 \% \mathrm{O}_{2}$ for $48 \mathrm{~h}$ in replete $(10 \% \mathrm{FBS}, 5 \mathrm{mM} \mathrm{glucose}), \mathrm{S}(0.5 \%$ FBS, $5 \mathrm{mM}$ glucose), and SG $\left(0.5 \%\right.$ FBS, $0.5 \mathrm{mM}$ glucose) media and then cultured for seven additional days in replete medium at $21 \% \mathrm{O}_{2}$.

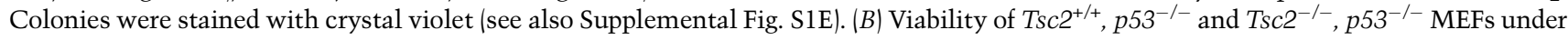
stress was also determined by exposing cells to $21 \%$ or $0.5 \% \mathrm{O}_{2}$ for $48 \mathrm{~h}$ in replete, $\mathrm{S}$, or SG medium, and cell survival was analyzed by flow cytometry $(P<0.001)$ (see also Supplemental Fig. S1A,B,F). $(C)$ The mTORC1 dependence of the survival phenotype was confirmed by rescuing $T s c 2^{-/-}, p 53^{-/-}$MEF cell death under SO limitation with $20 \mathrm{nM}$ rapamycin and $250 \mathrm{nM}$ torin (see also Supplemental Fig. S1C,H). (D) Viability of Tsc2 ${ }^{-1-}$ MEFs expressing wild-type TSC2 or an empty control vector was examined by exposing cells to replete and SO conditions for $48 \mathrm{~h}$. Cell survival was analyzed by flow cytometry $(P<0.001)$. (E) Pools of Tsc2 ${ }^{-1-}$, $p 53^{-1-}$ MEFs were depleted of raptor or rictor protein using siRNAs and cultured under SO conditions. The degree of knockdown as well as the effect on mTORC1 and AKT signaling was determined by probing for raptor and rictor protein abundance and for the phosphorylation status of S6K1, S6, and AKT by Western blot. $(F)$ Pools of Tsc2 $2^{-/-}, p 53^{-/-}$MEFs were depleted of raptor by siRNA treatment and cultured under SO conditions. After $48 \mathrm{~h}$, viability was assessed by flow cytometry $(P<0.001)$. (G) mTORC1, AKT, and MAPK signaling in Tsc2 $2^{+/+}, p 53^{-/-}$and Tsc2 ${ }^{-/-}$, $p 53^{-/-}$ MEFs under SO conditions for 0, 6, 12, 18, 24, and $30 \mathrm{~h}$ and SOG conditions for 0, 6, 12, 18, and $24 \mathrm{~h}$ was analyzed by blotting for the phosphorylation status of S6K1, S6, 4E-BP1, AKT, and p38 (see also Supplemental Fig. S1D,G). 
$\mathrm{Tsc}^{-/-}$MEFs transfected with either empty vector or a TSC2 expression construct (Ozcan et al. 2008) and determined that reintroduction of TSC2 increased cell survival (Fig. 1D). Furthermore, the effects of siRNAmediated knockdown of raptor (mTORC1-specific subunit) or rictor (mTORC2-specific subunit) on survival in $\mathrm{Tsc}^{-/-}$MEFs cultured under SO conditions were evaluated. Decreased raptor abundance and P-S6K1 levels verified efficacy of knockdown (Fig. 1E). Rictor inhibition was verified by both loss of expression and decreased levels of $\mathrm{P}$-AKT (Fig. 1E) and resulted in no change in $\mathrm{Tsc}^{-/-}$ viability under SO conditions (Fig. 1F). In contrast, knockdown of raptor partially rescued Tsc $2^{-/-}$cell viability (Fig. $1 F)$. Collectively, these data strongly indicate that constitutive mTORC1 activation promotes $\mathrm{Tsc}^{-/-}$cell death under SO conditions.

The mTORC1 dependence of ischemic cell death in $\mathrm{Tsc}^{-/-}, \mathrm{p} 53^{-/-}$MEFs prompted us to evaluate the effects of SO and SOG conditions on mTORC1 signaling preceding the appearance of apoptotic cells. We assessed two direct targets of mTORC1-S6K1 and 4E-BP1-that control distinct steps in the initiation of cap-dependent protein translation ( $\mathrm{Ma}$ and Blenis 2009) and P-AKT (Ser 473), a direct mTORC2 target. Exposing Tsc $2^{+/+}, p 53^{-/-}$ MEFs to SO or SOG conditions inhibited mTORC1 activity within $4 \mathrm{~h}$ (Supplemental Fig. S1D); in contrast, mTORC1 activity was sustained until $12-18 \mathrm{~h} \mathrm{in} \mathrm{Tsc}^{-{ }^{-/}}$, $\mathrm{p5}^{-/-} \mathrm{MEFs}$, as indicated by persistent S6K1, 4E-BP1, and S6 phosphorylation (Fig. 1G). Importantly, treatment with rapamycin inhibited $T s c 2^{-/-}, p 53^{-/-}$cell death (Fig. $1 C_{\text {; }}$ Supplemental Fig. S1C) and further shifted S6K1, S6, and 4E-BP1 to hypophosphorylated forms (Supplemental Fig. S1D). A decrease in the levels of P-AKT (Ser 473) and total AKT was observed in Tsc2 $2^{-/-}, p 53^{-/-}$compared with $\mathrm{Tsc}^{+/+}, p 53^{-/-}$MEFs under SO conditions; however, even a significant reduction in P-AKT signaling in Tsc2 $2^{-/-}, p 53^{-/-}$ MEFs did not further reduce cell survival (Fig. 1E,F). Under SOG conditions, a reduction in P-AKT activity in Tsc2 ${ }^{-/-}, p 53^{-/-}$MEFs was noted at 18 and $24 \mathrm{~h}$ (Fig. 1G). Because p38 MAP kinase is activated by a variety of stresses and promotes apoptosis, we examined p38 activation under SO and SOG conditions by evaluating phosphorylation at Thr 180/ Tyr 182. Slightly higher levels of P-p38 were noted in Tsc $2^{-/-}$, $p 53^{-/-}$MEFs under SO but not SOG conditions. Collectively, these results indicate that tumor-like stress causes a rapid reduction in mTORC1 activity in $\mathrm{Tsc}^{+/+}$, $p 53^{-/-}$MEFs, whereas this response is delayed in $\mathrm{Tsc}^{-/-}$, p53 ${ }^{-/-}$MEFs. mTOR is not the only pathway impacted in Tsc2 ${ }^{-/-}, p 53^{-/-}$MEFs affected by nutrient stress (Fig. 1G). However, because mTORC1 pharmacological inhibition, rescue of TSC2 expression, and raptor inhibition promoted $\mathrm{Tsc}^{-/-}, \mathrm{p} 53^{-/-}$cell viability under tumor-like stress, we concluded that dysregulated mTORC1 activity is an important component of ischemic cell death.

Tumor-like stress induces mTORC1-dependent cell death of T-antigen-immortalized MEFs

As our experiments were performed using $T s c 2^{-/-}, p 53^{-/-}$ MEFs with dysregulated mTORC1 activity, we investigated whether immortalized MEFs with a distinct genotype would exhibit similar responses to tumor-like stress conditions. Wild-type MEFs immortalized with SV40 large T-antigen attained a greater density in the colonyforming assay but also failed to survive under SO and SOG conditions (Supplemental Fig. S1E,F). Furthermore, we observed delayed attenuation of $\mathrm{mTORC1}$ activity in T-antigen-immortalized MEFs exposed to SO conditions, as evidenced by hypophosphorylation of P-S6 and 4E-BP1 (Supplemental Fig. S1G). Moreover, death of T-antigenimmortalized MEFs under SO and SOG conditions was suppressed by treatment with rapamycin (Supplemental Fig. $\mathrm{S} 1 \mathrm{H})$. These data indicate that our results are not peculiar to $T s c 2^{-/-}, p 53^{-/-}$MEFs but are also observed in immortalized MEFs with disparate genetic backgrounds.

\section{Tsc2 $2^{-/-}$, $5^{-/-}$MEFs maintain intracellular}

bioenergetics under serum and $\mathrm{O}_{2}$ limitation

Normal mammalian cells experiencing ischemic stress inhibit mTORC1 to limit mRNA translation and other ATP-consuming processes. We reasoned that constitutive mTORC1 activation could produce an intracellular energetic crisis associated with ATP depletion and consequent death in cells exposed to tumor-like stresses. To

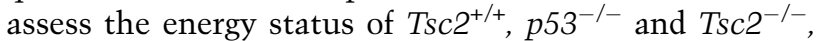
$p 53^{-1-}$ MEFs under SO and SOG conditions, we first investigated the activity of $\mathrm{AMPK}$, which responds to decreased ATP levels by suppressing cell growth and biosynthetic processes. We monitored phosphorylation of AMPK on Thr 172, an activating event mediated by LKB1 as an indicator of cellular energetic stress. As a positive control, Tsc2 $2^{+/}, p 53^{-/-}$and Tsc2 $2^{-/-}, p 53^{-/-}$MEFs were treated with $1 \mathrm{mM}$ 5-aminoimidazole-4-carboxyamide ribonucleoside (AICAR) to stimulate AMPK activity.

Exposure to $30 \mathrm{~h}$ of SOG conditions resulted in AMPK

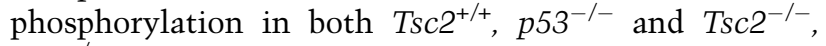
p53 ${ }^{-/-}$MEFs (Fig. 2A). Levels of AMPK phosphorylation in $\mathrm{Tsc}^{+/+}, \mathrm{p} 53^{-/-}$and $\mathrm{Tsc} 2^{-/-}, \mathrm{p} 53^{-/-}$MEFs can be directly compared in Supplemental Figure S2A. Interestingly, the combination of serum and $\mathrm{O}_{2}$ deprivation alone did not activate AMPK in Tsc2 $2^{-/-}, p 53^{-/-}$MEFs (Fig. 2A), suggesting that SO conditions induce cell death without generating an energetic crisis. Furthermore, direct measurement revealed that intracellular ATP concentrations were maintained in both SO-treated $\mathrm{Tsc}^{+/+}, \mathrm{p} 53^{-/-}$and Tsc2 $2^{-/-}, p 53^{-/-}$MEFs, compared with "O" $\left(0.5 \% \mathrm{O}_{2}\right.$, $10 \%$ serum) conditions (Fig. 2B). As expected, ATP levels were significantly reduced in $T s c 2^{+/+}, p 53^{-/-}$and $\mathrm{Tsc}^{-/-}$, $p 53^{-1-}$ MEFs under SOG conditions. Moreover, the caspase inhibitor Z-VAD-FMK rescued the viability of $\mathrm{Tsc}^{-1-}, \mathrm{p} 53^{-/-}$MEFs under SO conditions (Fig. 2C). Collectively, these data indicate that MEFs with dysregulated mTORC1 activity undergo apoptosis when challenged with combined serum and $\mathrm{O}_{2}$ limitation despite exhibiting normal levels of glucose consumption (Supplemental Fig. S2B) and intracellular ATP. It is interesting to note that cell survival was significantly higher in $\mathrm{Tsc}^{+/+}, \mathrm{p} 53^{-/-}$MEFs, compared with $\mathrm{Tsc} 2^{-/-}, \mathrm{p} 53^{-/-}$ MEFs under SOG conditions (Fig. 1B), although both cell 
A
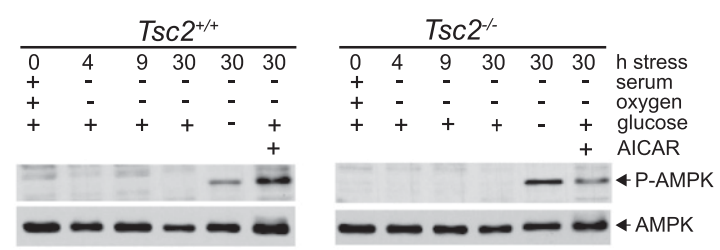

B
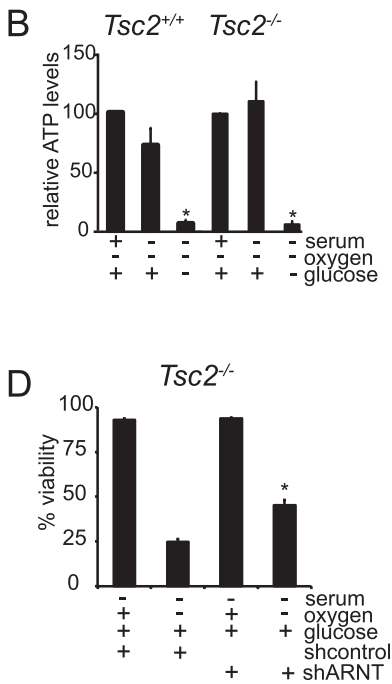

$\mathrm{F}$
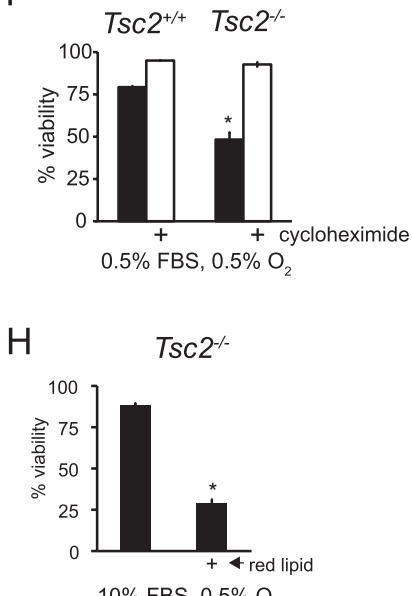

$10 \%$ FBS, $0.5 \% \mathrm{O}_{2}$

lines exhibited low intracellular ATP levels. These data suggest that limiting ATP is not the sole factor regulating apoptosis in $T s c 2^{-/-}, p 53^{-/-}$MEFs under these conditions.

$\mathrm{HIF}-1 \alpha$ promotes metabolic adaptations under low $\mathrm{O}_{2}$, such as increased glucose uptake and glycoysis, and HIF$1 \alpha$ expression is enhanced in an mTORC1-dependent manner; therefore, we investigated whether the viability of Tsc2 $^{-/-}, p 53^{-/-}$MEFs exposed to tumor-like stress was influenced by HIF activity. We inhibited ARNT (HIF-1 $\alpha$ dimerization partner) in Tsc2-null cells using an Arnt shRNA and verified the efficiency of knockdown by Western (Supplemental Fig. S2C). When the viability of Tsc2-null and Tsc2-null, ARNT knockdown MEFs was compared under SO conditions, the ARNT-depleted MEFs exhibited statistically significant less cell death
Figure 2. $T s c 2^{-/-}, p 53^{-/-} \mathrm{MEFs}$ maintain intracellular bioenergetics under serum and $\mathrm{O}_{2}$ limitation. $(A)$

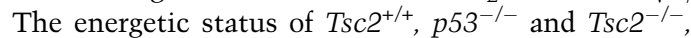
$p 53^{-1-}$ MEFs in SO conditions was evaluated by assaying the phosphorylation status of AMPK (Thr 172) at $0,4,9$, and $30 \mathrm{~h}$ and in the presence of $1 \mathrm{mM}$ AICAR or SOG medium for $30 \mathrm{~h}$. (B) Relative ATP levels were determined in $T s c 2^{+/+}, p 53^{-/-}$and $T s c 2^{-/-}$, $p 53^{-/-}$MEFs exposed to O, SO, and SOG conditions for $18 \mathrm{~h}(P<0.001)$ (see also Supplemental Fig. S2A,B). (C) Tsc2 $2^{-/}$, $p 53^{-/-}$MEF cell death under SO conditions is rescued by $100 \mu \mathrm{M} \mathrm{Z-VAD-FMK,} \mathrm{which}$ inhibits caspases $(P<0.001)$. $(D) \mathrm{Tsc}^{+/+}, \mathrm{p} 53^{-/-}$and Tsc2 $2^{-/-}, p 53^{-/-}$MEFs were depleted of ARNT protein by treatment with lentivirus expressing shRNAs (see also Supplemental Fig. 2SC). Cells were cultured under SO conditions for $48 \mathrm{~h}$, and viability was evaluated by flow cytometry $(P<0.001)$. $(E) \mathrm{Tsc}^{+/+}$, $p 53^{-/-}$and $\mathrm{Tsc} 2^{-/-}, \mathrm{p} 53^{-/-}$MEFs were depleted of HIF- $1 \alpha$ protein by treatment with lentivirus expressing shRNAs $(P<0.005)$ (see also Supplemental Fig. 2SD). Cells were cultured under SO conditions for $48 \mathrm{~h}$, and viability was evaluated by flow cytometry. $(F)$ To determine whether cycloheximide rescues cell death under SO limitation, $\mathrm{Tsc}^{+/+}, \mathrm{p} 53^{-/-}$and $T s c 2^{-/-}, p 53^{-/-}$MEFs were exposed to SO conditions with or without $1 \mu \mathrm{M}$ cycloheximide, and cell viability was assayed $(P<0.001)$. $(G)$ Representative electron micrographs illustrate the differences in ER morphology between $\mathrm{Tsc}^{+/+}, \mathrm{p} 53^{-/-}$and $\mathrm{Tsc}^{-/-}$, $p 53^{-/-}$MEFs cultured under SO limitation. White arrows highlight the ER, while black arrows indicate autophagosomes (see also Supplemental Fig. S2E). $(H)$ We compared the viability of $T s c 2^{-1-}, p 53^{-/-}$MEFs exposed to $10 \%$ FBS or $10 \%$ lipid depleted FBS Ored $(P<0.001)$ ( see also Supplemental Fig. S2F).
(Fig. 2D). This result was confirmed by comparing Tsc2null control and HIF-1 $\alpha$ knockdown MEF viability under SO conditions (Fig. 2E; Supplemental Fig. S2D). Again, Tsc2-null, HIF-1 $\alpha$ knockdown MEFs exhibited increased viability compared with controls. We concluded that cell death under SO conditions is at least partially HIF-dependent. This is not surprising given that HIF- $1 \alpha$ promotes metabolic adaptations under low $\mathrm{O}_{2}$, such as increased glucose uptake, elevated glycolysis, and limited flux through the TCA cycle, resulting in decreased production of acetyl-CoA, a precursor for lipid synthesis.

As constitutive mTORC1 activity can promote inappropriate protein translation, leading to an overload of misfolded proteins in the ER (Ozcan et al. 2008), we considered the possibility that unresolved ER stress in 
Tsc2 ${ }^{-/-}, p 53^{-/-}$MEFs might sensitize cells to tumor-like stress. Consistent with this notion, the viability of SOtreated $T s c 2^{-/-}, p 53^{-/-}$MEFs was rescued by treatment with the protein synthesis inhibitor cycloheximide (Fig. $2 \mathrm{~F})$. Low levels of cycloheximide $(1 \mu \mathrm{M})$ rescued cell viability in Tsc2 $2^{-/-}, p 53^{-/-}$MEFs exposed to SO conditions without altering the cell cycle profile. After $24 \mathrm{~h}$, $57.5 \%$ of Tsc2-deficient MEFs were in G1 without cycloheximide treatment, and $55.3 \%$ were in G1 with cycloheximide treatment (three independent experiments) (data not shown). These results suggest a direct connection between unregulated protein synthesis and cell death. Furthermore, we examined the ultrastructure of control and Tsc2-deficient MEFs, and representative electron micrographs of cells from each genotype are shown in Figure 2G and Supplemental Figure S2E. Normal ER ultrastructure was observed in both $T s c 2^{+/+}, p 53^{-/-}$and $\mathrm{Tsc}^{-/-}, \mathrm{p} 53^{-/-}$MEFs under replete conditions (Supplemental Fig. S2E). However, under SO conditions, unlike $\mathrm{Tsc}^{+/+}$, p53 $3^{-/-}$cells, Tsc2-null MEFs show widely distended intracellular membranes, due in part to structural changes in the ER, since ribosomes are sometimes associated with the swollen membranes (Fig. 6E, below). In support of these data, Diakogiannaki et al. (2008) observed similar morphological alterations in ER within pancreatic $\beta$ cells exhibiting definitive evidence of ER stress. Surprisingly, we also noted the presence of autophagosomes in micrographs of $T s c 2^{-/-}, p 53^{-/-}$MEFs cultured under SO conditions (Fig. 2G, panel d, black arrows), discussed below.

In addition to enhancing protein synthesis and HIFdependent glycolysis, dysregulated mTORC1 activity is known to promote de novo lipid synthesis (Duvel et al. 2010), suggesting that mTORC1-driven lipid synthesis might be impaired under nutrient limitation and contribute to the death of Tsc2-null MEFs. In particular, because hypoxic Tsc2-null MEFs are viable in normal serum but exhibit loss of viability when serum is limited (Fig. 1A,B), we wondered whether $T s c 2^{-1-}$ cells required lipids supplied in the serum for viability under low $\mathrm{O}_{2}$. To test this hypothesis, we compared the survival of hypoxic $\mathrm{Tsc}^{-/-}$, $p 53^{-1-}$ MEFs cultured in normal serum and lipid-reduced serum $\left(0.5 \% \mathrm{O}_{2}, 10 \%\right.$ lipid-reduced serum, designated "Ored") and observed that Tsc2-null cells were viable in normal serum but exhibited loss of viability when lipids were removed (Fig. 2H). Similar results were obtained with T-antigen-immortalized MEFs, which remained viable under $S$ conditions but exhibited cell death under $S$ conditions when normal serum was replaced with lipiddepleted serum (Supplemental Fig. S2F). These results indicate that viability of Tsc2-null cells exposed to SO conditions is impacted by both protein and lipid synthesis and demonstrate that exogenous lipids are a critically limiting nutrient when cells with constitutive mTORC1 activity are deprived of serum and $\mathrm{O}_{2}$.

Unsaturated fatty acids rescue Tsc2 $2^{-1-}$ cell death under tumor-like stress

We next investigated which serum lipids were specifically required for maintaining the viability of $\mathrm{Tsc}^{-/-}$ cells exposed to SO or Ored stress. Addition of unsaturated fatty acids, such as a combination of oleic (18:1) and (18:2) linoleic acids, rescued cell death under both SO or Ored conditions (Fig. 3A,B); however, addition of saturated (16:0) palmitic acid did not (Fig. 3A,B). We examined the ability of $35 \mu \mathrm{M}$ oleic, palmitic, octanoic, and hexanoic acid to rescue the viability of Tsc2-null MEFs under SO conditions and again observed that unsaturated, but not saturated, fatty acids restored Tsc2-null cell survival (Fig. 3C). These data suggest that unsaturated fatty acids, which serve as precursors for signaling lipids and membrane biosynthesis, are critically limiting in $T s c 2^{-/-}, \mathrm{p}^{-/-}$ MEFs under SO conditions. Oleic acid or oleic/linoleic acid also partially rescued the viability of $T s c 2^{-/-}, p 53^{-/-}$MEFs under SOG conditions (Fig. 3D), although reduced ATP levels (Fig. 2B) likely also contribute to cell death (Fig. 1B).

The effect of lipid-replete and -deficient FBS and oleic or palmitic acid supplementation on mTOR signaling was assayed by determining the phosphorylation status of mTOR effectors AKT (Ser 473), S6K1, and 4E-BP1 (Fig. $3 E)$. We observed a subtle activation of mTORC1 signaling in Tsc2-null MEFs under SO and Ored conditions with the addition of oleic acid and, conversely, mTOR signaling inhibition with the addition of palmitic acid (Fig. 3E). These findings indicate that MTORC1 activity is somewhat influenced by the availability of unsaturated and saturated fatty acids. However, oleic acid rescue of $T s c 2^{-/-}$ cell viability under SO conditions is not mediated by mTORC1 inhibition.

As the enzyme stearoyl-CoA desaturase-1 (SCD1) generates monounsaturated fatty acids from saturated fatty acids, we assayed the levels of Scd1 expression in Tsc2 $2^{-/-}$ cells and found that Scd1 mRNA levels were actually elevated under multiple stress conditions (S, SO, and SOG) (Fig. 3F) as compared with replete conditions, verifying that reduced Scd1 mRNA levels do not account for decreased lipid desaturation in these cells.

\section{Desaturation of de novo synthesized lipids is reduced under low $\mathrm{O}_{2}$}

We examined the pattern of de novo lipogenesis in Tsc2 $2^{-1-}$ cells by nuclear magnetic resonance (NMR) spectroscopy. Tsc2 $2^{-/-}$, $p 53^{-1-}$ MEFs were grown under $21 \%$ or $0.5 \% \mathrm{O}_{2}$ in medium containing either $10 \mathrm{mM}\left[\mathrm{U}^{13} \mathrm{C} 6\right]$ glucose or $3 \mathrm{mM}\left[5-{ }^{13} \mathrm{C}\right]$ glutamine for $24 \mathrm{~h}$, and the lipid spectra are displayed in Supplemental Figure S3, A and B. The contribution of serum-derived lipids to lipid synthesis in $\mathrm{Tsc}^{-/-}$MEFs was determined by taking advantage of the natural abundance of ${ }^{13} \mathrm{C}$. Data from the NMR spectra are represented in histogram form (Fig. 3G). As expected, the contribution of glucose to de novo lipogenesis decreased under hypoxia $\left(21 \% \mathrm{O}_{2}, 14.4 \% ; 0.5 \% \mathrm{O}_{2}, 7.2 \%\right)$; conversely, the contribution of glutamine-derived carbon to de novo lipogenesis increased under hypoxia $\left(21 \% \mathrm{O}_{2}, 8.0 \%\right.$; $\left.0.5 \% \mathrm{O}_{2}, 14.4 \%\right)$, resulting in nearly unchanged total levels of de novo lipogenesis from glucose and glutamine. These results directly support recently published data that examine the role of reductive glutamine metabolism in lipogenesis under hypoxia (Metallo et al. 2011; Wise et al. 
A

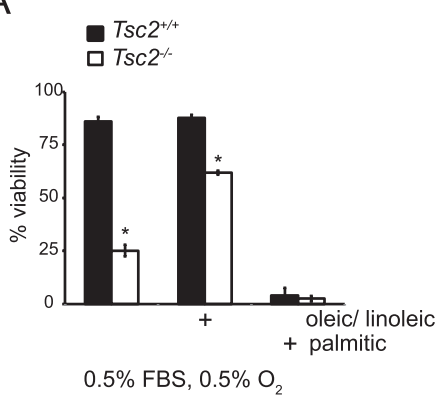

C

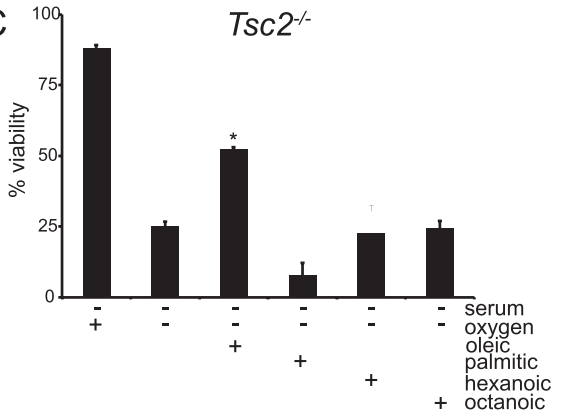

B

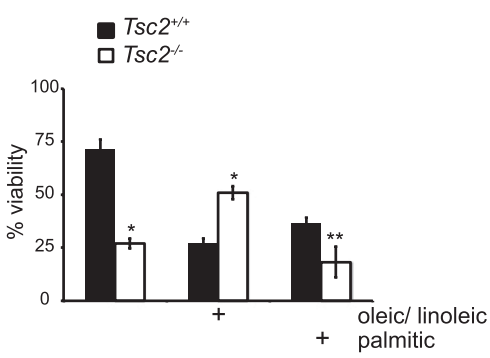

$10 \%$ lipid reduced $\mathrm{FBS}, 0.5 \% \mathrm{O}$

D

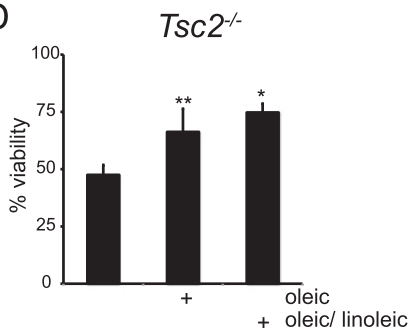

$0.5 \%$ FBS, $0.5 \% \mathrm{O}_{2}, 0.5 \mathrm{mM}$ glucose
E

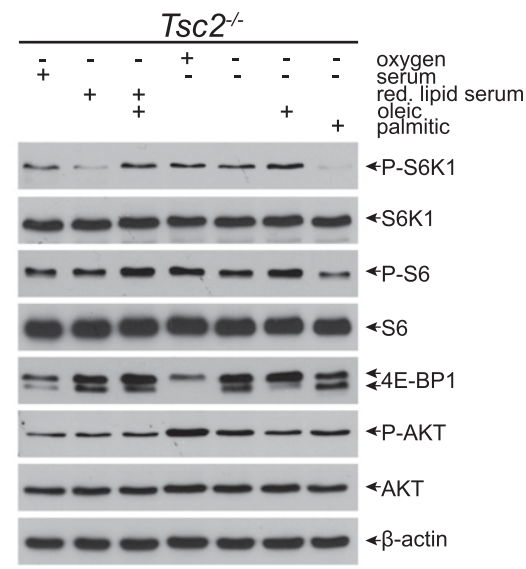

$\mathrm{F}$

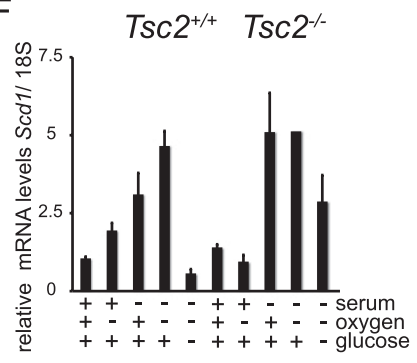

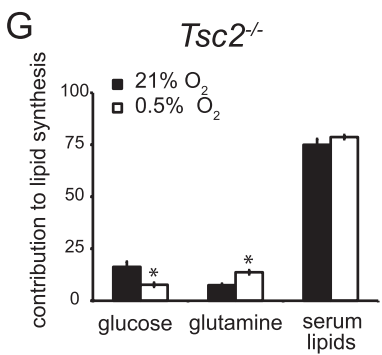
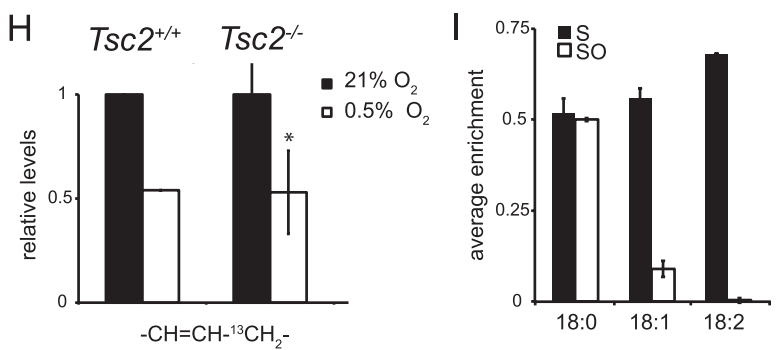

Figure 3. Unsaturated fatty acids rescue $T s c 2^{-/-}$cell death under tumor-like stress. $(A)$ The ability of unsaturated fatty acids to rescue $\mathrm{Tsc}^{-/-}, p 53^{-/-}$cell death under tumor-like stress was assessed by culturing MEFs under SO conditions for $48 \mathrm{~h}$ with or without $50 \mu \mathrm{M}$ oleic/linoleic acid or palmitic acid $(P<0.001) .(B) T s c 2^{-/-}, p 53^{-/-}$MEFs were cultured for $48 \mathrm{~h}$ under Ored conditions in the presence and absence of $50 \mu \mathrm{M}$ oleic/linoleic or palmitic acid $(P<0.001)$. $(C)$ The ability of $35 \mu \mathrm{M}$ oleic, palmitic, hexanoic, or octanoic acid to rescue Tsc2 ${ }^{-/-}$cell death under $48 \mathrm{~h}$ of SO stress was determined by flow cytometry. $\left(^{\star \star}\right) P<0.005$; $\left(^{\star}\right) P<0.001$. (D) The ability of $50 \mu \mathrm{M}$ oleic acid or oleic/linoleic acid to rescue $T s c 2^{-/-}$cell death after $48 \mathrm{~h}$ of SOG stress was determined by flow cytometry. $\left.\left(^{\star \star}\right) P<0.005 ;{ }^{\star}{ }^{\star}\right) P<$ 0.001. (E) mTORC1 and AKT signaling was assessed in response to addition of oleic or palmitic acid under lipid-deficient conditions. mTORC1 and AKT signaling in Tsc2 $2^{+/}, p 53^{-/-}$and $T s c 2^{-/-}, p 53^{-/-}$MEFs was analyzed by blotting for the phosphorylation status of S6K1,

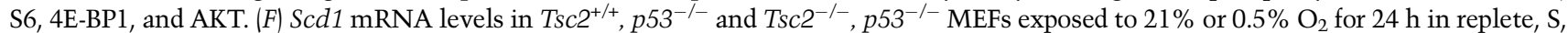
or SG medium were determined by qRT-PCR. $(G) \mathrm{Tsc}^{+/+}, p 53^{-/-}$and $\mathrm{Tsc}^{-/-}, p 53^{-/-}$MEFs were grown under $21 \%$ or $0.5 \% \mathrm{O}_{2}$ in medium containing $10 \mathrm{mM}\left[\mathrm{U} \cdot{ }^{13} \mathrm{C} 6\right]$ glucose or $3 \mathrm{mM}\left[5-{ }^{13} \mathrm{C}\right]$ glutamine for $24 \mathrm{~h}$, and the relative contribution of glucose, glutamine, and serum-derived lipids to lipid synthesis in Tsc2 $2^{-/}$MEFs was determined from the NMR chemical spectra (see also Supplemental Fig. S3A,B). These results are displayed in histogram form $(P<0.005)$. $(H)$ The relative levels of de novo unsaturated fatty acids in $T s c 2^{+/+}$, $p 53^{-/-}$and $\mathrm{Tsc}^{-{ }^{--}}, \mathrm{p} 53^{-/-}$MEFs cultured under normoxic $\left(21 \% \mathrm{O}_{2}\right)$ and hypoxic $\left(0.5 \% \mathrm{O}_{2}\right)$ conditions were calculated from the NMR chemical spectra and are presented as a bar graph $(P<0.001)$. (I) The relative levels of newly synthesized stearic, oleic, and linoleic acid in $T s c 2^{-/-}, p 53^{-/-}$MEFs cultured under S and SO conditions as well as under SO conditions were assessed by GC-MS. 
Young et al.

2011). Unlabeled fatty acids in the serum accounted for $\sim 80 \%$ of total cellular lipid synthesis in Tsc2 $2^{-/-}, p 53^{-/-}$ MEFs under both $21 \%$ and $0.5 \% \mathrm{O}_{2}$, which was surprising in light of reports delineating critical roles for de novo lipid biosynthesis in cancer cell growth (Menendez and Lupu 2007). Interestingly, desaturation of de novo synthesized lipids was decreased by $50 \%$ in both $\mathrm{Tsc}^{+/+}$and $\mathrm{Tsc}^{-/-}$ cells exposed to $0.5 \% \mathrm{O}_{2}$ as compared with $21 \% \mathrm{O}_{2}($ Fig. $3 \mathrm{H})$, indicating that lipid desaturation is strongly inhibited by hypoxia and that $T s c 2^{-1-}$ cells are more sensitive than $\mathrm{TsC2}^{+/+}$cells to reduced levels of desaturated lipids. We confirmed and extended these results by analyzing the composition of total and de novo synthesized fatty acids under $\mathrm{S}$ and $\mathrm{SO}$ conditions by gas chromatography-mass spectrometry (GC-MS) (Fig. 3I; Supplemental Fig. S3C). Representative chromatograms of lipid extracts obtained from $T s c 2^{-/}$, $p 53^{-/-}$MEFs cultured under the various conditions are displayed in Supplemental Figure S3C. SO conditions elicited lower levels of unsaturated compared with saturated fatty acids (16 and $18 \mathrm{C}$ fatty acids shown), suggesting that availability of unsaturated fat, either from serum or synthesized de novo, is reduced. Furthermore, the levels of newly synthesized lipids, as indicated by the average enrichment from ${ }^{13} \mathrm{C}$ glucose, were nearly equal for 18:0, 18:1, and 18:2 fatty acids under $S$ conditions.
However, under SO conditions, de novo synthesis of unsaturated fats was markedly reduced (Fig. 3I). These results demonstrate that both total and de novo synthesized unsaturated, but not saturated, fatty acids are reduced under SO conditions.

\section{Restoration of autophagic flux fails to rescue Tsc2 ${ }^{-/-}$, p53 ${ }^{-/-}$cell viability under SO conditions}

Because mTORC1 activity is known to inhibit autophagy, yet autophagosomes were readily apparent in SOtreated Tsc2 $2^{-/-}$MEFs (Fig. 2G), we investigated autophagic signaling in $\mathrm{Tsc}^{-/-}$cells under SO conditions. Specifically, we examined the expression of autophagy effectors lipidated LC3 (LC3II) and p62 in control and Tsc2 ${ }^{-/-}$MEFs under various stress conditions (Fig. 4A). The levels of LC3II and p62 in Tsc2 $2^{-/-}$, $p 53^{-/-}$MEFs under SO conditions were comparable with those achieved with bafilomycin treatment, consistent with significantly reduced autophagic flux and increased levels of autophagosomes observed in these cells. This block in autophagy was resolved by the addition of oleic acid, which reduced the expression of LC3II and p62 as well as the UPR marker CHOP (Fig. 4A). Bafilomycin blocks the oleic acid rescue, confirming that oleic acid is restoring autophagic flux

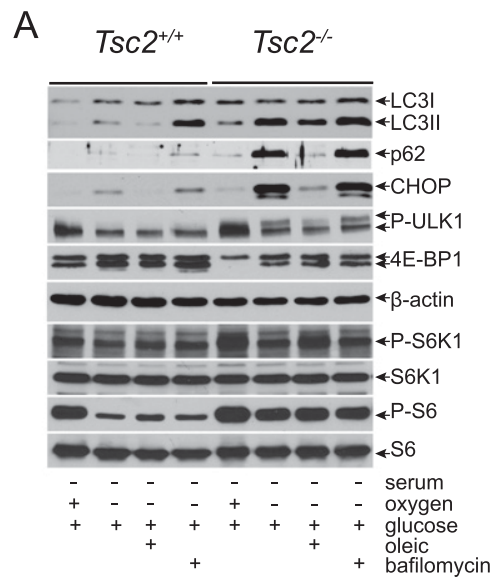

C

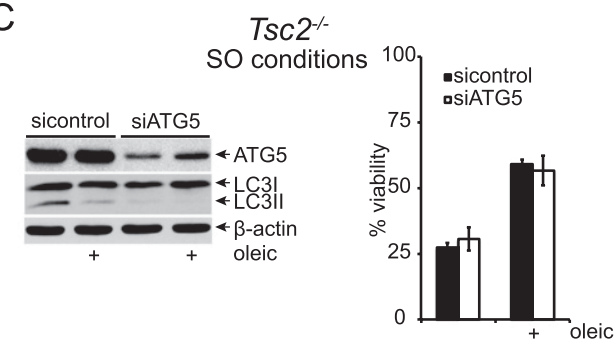

B

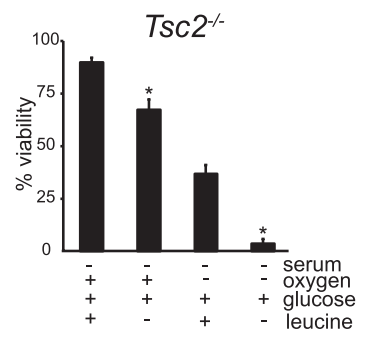

D

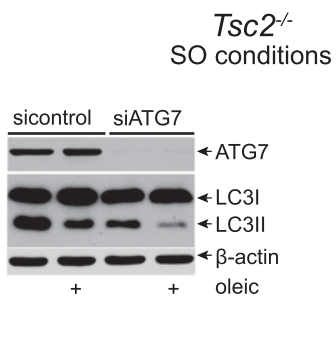

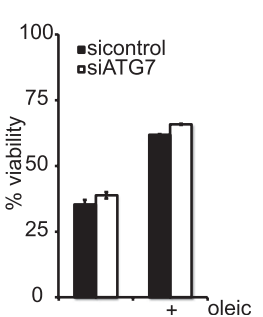

Figure 4. Restoration of autophagic flux fails to rescue $T s c 2^{-/-}, p 53^{-/-}$cell viability under SO conditions. (A) Autophagic signaling and flux were examined in Tsc2 ${ }^{+/+}, p 53^{-/-}$and $\mathrm{Tsc}^{-/-}, \mathrm{p} 53^{-/-}$MEFs under S and SO conditions for $24 \mathrm{~h}$ by assaying the levels of LC3I, LC3II, p62, and CHOP with and without $100 \mathrm{nM}$ bafilomycin. mTORC1 signaling was monitored by assessing the phosphorylation status of ULK1, 4E-BP1, S6K1, and S6. (B) The effect of leucine deprivation on viability under $48 \mathrm{~h}$ of S and SO conditions was examined $(P<0.001) .(C, D)$ The contribution of autophagy to oleic acid rescue of $T s c 2^{-/-}, p 53^{-/-}$MEF cell death under SO conditions was assayed. Pools of Tsc2 ${ }^{-1-}, p 53^{-/-}$MEFs were depleted of ATG5 $(C)$ or ATG7 $(D)$ protein using siRNAs and cultured under SO conditions in the presence and absence of oleic acid. After $48 \mathrm{~h}$, viability was assessed by flow cytometry. The degree of knockdown was determined by Western blot. 
(Supplemental Fig. S4). Interestingly, oleic acid did not restore autophagic flux by down-regulating mTORC1 activity in Tsc2 $2^{-/-}, p 53^{-/-}$MEFs under SO conditions, as no dramatic changes in ULK1, 4E-BP1, S6K1, or S6 phosphorylation were observed upon unsaturated fatty acid supplementation (Fig. 4A). Because cells defective in autophagy are sensitive to leucine deprivation (Sheen et al. 2011), we examined the viability of $T s c 2^{-/-}$cells cultured under S and SO conditions in the presence and absence of leucine (Fig. 4B). Of note, increased cell death was observed upon leucine depletion, confirming their autophagic status.

To test whether oleic acid could rescue $\mathrm{Tsc2}^{-/-}$cell viability under SO conditions by restoring autophagic flux, we used siRNAs to knock down the expression of the essential autophagy components ATG5 or ATG7. Inhibition of both ATG5 and ATG7 resulted in decreased levels of LC3II but did not affect the ability of oleic acid to rescue $T s c 2^{-/-}$cell viability under SO conditions (Fig. $4 \mathrm{C}, \mathrm{D}$ ), demonstrating that the restoration of autophagic flux by desaturated lipids is not sufficient to explain the rescue of $\mathrm{Tsc}^{-/-}$cell viability under tumor-like stress.

Dysregulated mTORC1 activity promotes a magnified $E R$ stress response under nutrient and $\mathrm{O}_{2}$ limitation

Since dysregulated protein synthesis and reduced lipid desaturation contribute to the death of $T s c 2^{-/-}, p 53^{-/-}$ MEFs and both processes are associated with the ER, we hypothesized that SO and SOG conditions produce severe ER stress in these cells and induce apoptosis through consequent engagement of the UPR (Ozcan et al. 2008). To examine UPR activation under tumor-like stress, we analyzed cell extracts from $T s c 2^{+/}, p 53^{-/-}$and $T s c 2^{-/-}$, $p 53^{-1-}$ MEFs for activation of PERK and IRE1 $\alpha$ and the accumulation of their respective downstream targets, $\mathrm{CHOP}$ and $\mathrm{XBP} 1_{\mathrm{s}}$ (Fig. 5A). We found that PERK autophosphorylation, a marker of PERK activation, was most reliably assessed by an upward mobility shift in immunoblots using an antibody directed against total PERK protein (Supplemental Fig. S5A). PERK was activated in serum-deprived $\mathrm{Tsc}^{-/-}, \mathrm{p} 53^{-/-} \mathrm{MEFs}$, and this response was magnified under SO and SOG conditions (Fig. 5A). Furthermore, a significant accumulation of CHOP was observed in Tsc2 $2^{-/-}, p 53^{-/-}$MEFs under both SO and SOG stresses (Fig. 5A). Interestingly, we noted a highly selective induction in IRE1 $\alpha$ phosphorylation and accumulation of spliced X-box-binding protein $1\left(\mathrm{XBP}_{\mathrm{s}}\right)$ in $\mathrm{Tsc} 2^{-/-}, \mathrm{p} 53^{-/-}$ MEFs under SO conditions at $24 \mathrm{~h}$. In contrast, IRE $1 \alpha$ activation, as measured by the accumulation of $\mathrm{XBP} 1_{\mathrm{s}}$ peaked by $12 \mathrm{~h}$ under SOG limitation (Supplemental Fig. S5B). Similarly, we observed a magnification of PERK autophosphorylation and increased accumulation of $\mathrm{XBP}_{\mathrm{s}}$ and $\mathrm{CHOP}$ in T-antigen-immortalized MEFs under SO and SOG conditions (Supplemental Fig. S5C). Treatment with rapamycin reversed the activation of PERK as well as the accumulation of $\mathrm{XBP} 1_{\mathrm{s}}$ and $\mathrm{CHOP}$, confirming the mTORC1 dependence of UPR activation in $\mathrm{Tsc}^{-/-}$, $p 53^{-/-}$and T-antigen- immortalized MEFs exposed to tumor-like stress.
As ischemic death of $T s c 2^{-/-}, p 53^{-/-}$MEFs was observed only at $0.5 \% \mathrm{O}_{2}$ (Fig. 1A), we examined UPR signaling in $\mathrm{Tsc}^{-/-}, \mathrm{p} 53^{-/-}$MEFs under a range of $\mathrm{O}_{2}$ levels with or without serum limitation. Under low serum, gradual increases in PERK autophosphorylation and CHOP accumulation were observed as $\mathrm{O}_{2}$ levels dropped from $21 \%$ to $0.5 \% \mathrm{O}_{2}$ (Supplemental Fig. S5D). In contrast, IRE1 $\alpha$ was specifically phosphorylated in $T s c 2^{-/-}, p 53^{-/-}$MEFs only at $0.5 \% \mathrm{FBS}$ and $0.5 \% \mathrm{O}_{2}$ but not at $21 \%, 3 \%$, or $1.5 \% \mathrm{O}_{2}$ (Supplemental Fig. S5D), implicating IRE1 $\alpha$ activation in mTORC1-mediated cell death. UPR activation increases the expression of mRNA transcripts from multiple genes, including heme oxygenase 1 (Ho-1), binding immunoglobulin protein (Bip), activating transcription factor 4 (Atf4), Xbp1 $1_{s}$, unspliced Xbp1 $\left(X b p 1_{u}\right)$, and homocysteineresponsive endoplasmic reticulum-resident ubiquitin-like domain member 1 protein (Herp). Quantitative RT-PCR (qRT-PCR) analysis revealed a significant induction of these transcripts in $T s c 2^{-/-}, p 53^{-/-}$cells but not $\mathrm{Tsc}^{+/+}$, $p 53^{-/-}$MEFs specifically under SO conditions at $16 \mathrm{~h}$ (Fig. 5B), which mirrors the activation of PERK and IRE1 $\alpha$. Collectively, these results indicate that the UPR is dramatically amplified, with attendant changes in ER ultrastructure, in response to combined microenvironmental stresses in Tsc2-null cells.

Our data reveal a correlation between inappropriate protein synthesis, decreased availability of desaturated lipids, and cell death in Tsc2-null cells under SO and SOG conditions. Since the reduction of serum lipids under hypoxia induced $T s c 2^{-1-}$ cell death (Fig. $2 \mathrm{H}$ ), we asked whether these conditions (Ored) were also sufficient to amplify the UPR. We observed a stepwise increase in the phosphorylation of PERK and IRE1 $\alpha$ and the accumulation of $\mathrm{CHOP}$ in $\mathrm{Tsc}^{-/-}$cells exposed to $\mathrm{O}$, Ored, and $\mathrm{SO}$ conditions for $24 \mathrm{~h}$ (Fig. 5C), supporting the correlation between UPR engagement and lipid availability in cells with dysregulated mTORC1 activity. We next asked whether unsaturated fatty acids could rescue the magnified UPR and attendant changes in ER ultrastructure observed in Tsc2-null cells in response to combined microenvironmental stresses. Addition of oleic acid to $\mathrm{Tsc}^{-/-}, p 53^{-/-}$MEFs under SO conditions diminished PERK and IRE $1 \alpha$ phosphorylation and CHOP abundance (Fig. 5D). Finally, we demonstrated that the aberrant ER ultrastructure in $\mathrm{Tsc}^{-/-} \mathrm{MEFs}$ cultured under SO conditions can be rescued by treatment with unsaturated fatty acids (Fig. 5E). These data suggest that direct supplementation of unsaturated fatty acids suppressed a lipid-dependent stress response, confirming the link between protein and lipid synthesis through UPR engagement downstream from ER stress.

All three arms of the UPR induce transcriptional programs that act to alleviate ER stress. However, when ER stress becomes too severe, the UPR can promote cell death. To determine whether UPR activation was necessary for $T s c 2^{-1-}, p 53^{-/-}$cell death, we evaluated the effects of PERK, CHOP, or IRE1 $\alpha$ inhibition on cell viability. PERK deletion (Supplemental Fig. S5E) or knockdown (Fig. 5F; Supplemental Fig. S5F) did not rescue viability under SO conditions, indicating that PERK does not 


\section{A}

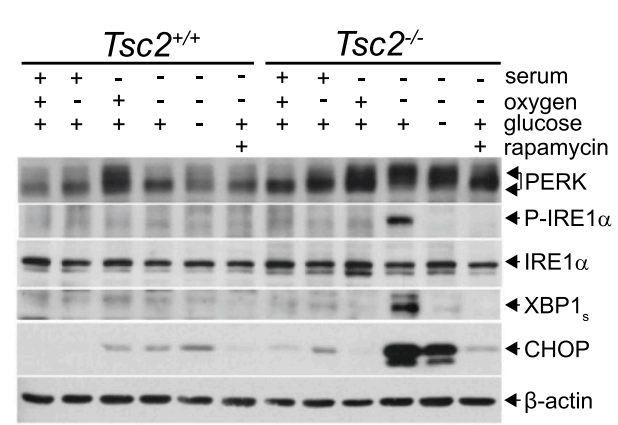

B

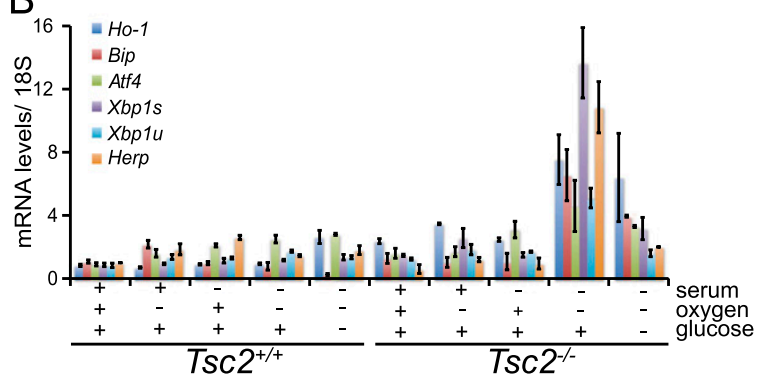

C

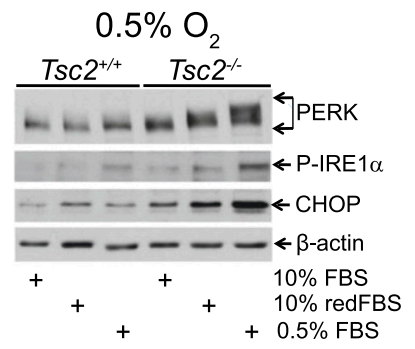

D Tsc2 ${ }^{-/-}$

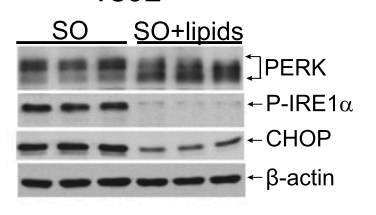

E

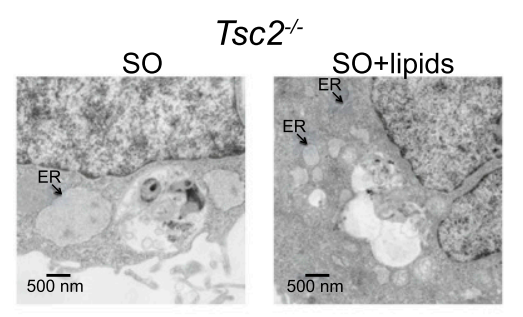

F

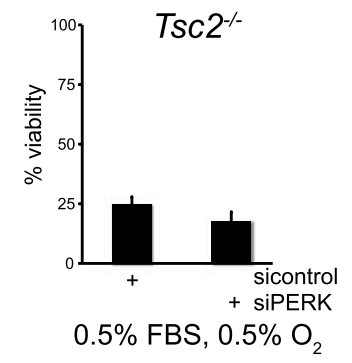

G

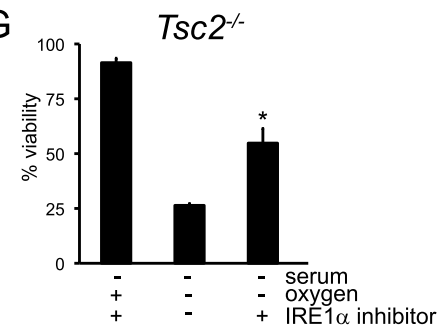

Figure 5. Dysregulated mTORC1 activity promotes a magnified ER stress response under nutrient and $\mathrm{O}_{2}$ limitation. $(A)$ To evaluate which sensors of the UPR are activated under ischemic stress, $T s c 2^{+/+}, p 53^{-/-}$and $\mathrm{Tsc}^{-/-}, \mathrm{p53^{-/- }}$ MEFs were exposed for $24 \mathrm{~h}$ to $21 \%$ or $0.5 \% \mathrm{O}_{2}$ in replete, $\mathrm{S}$, and SG conditions. Whole-cell extracts were blotted for PERK, P-IRE1 $\alpha$ (Ser 724), IRE1 $\alpha, \mathrm{XBP} 1_{\mathrm{s}}$ CHOP, and $\beta$-actin (see also Supplemental Fig. S5A-D). (B) Tsc2 ${ }^{+/+}, p 53^{-/-}$and $T s c 2^{-/-}$, $p 53^{-1-}$ MEFs were exposed to $21 \%$ and $0.5 \%$ $\mathrm{O}_{2}$ in replete, $\mathrm{S}$, or SG medium for $16 \mathrm{~h}$, and levels of UPR target mRNAs Ho-1, Bip, Atf4, $X b p 1_{s}, X b p 1_{u}$, and Herp were determined by qRT-PCR. (C) The autophosphorylation of PERK and IRE1 $\alpha$ and the induction of CHOP in $T s c 2^{+/+}, p 53^{-/-}$and Tsc2 $2^{-/-}, p 53^{-/-}$MEFs exposed to $\mathrm{O}$, Ored, and SO conditions were assayed by Western blot analysis. (D) To determine whether oleic acid alters UPR signaling pathways under tumor-like stress, $\mathrm{Tsc}^{-/-}, \mathrm{p} 53^{-/-}$MEFs were cultured under SO conditions in the presence or absence of $50 \mu \mathrm{M}$ oleic acid, and whole-cell extracts were blotted for PERK, P-IRE1 $\alpha$ (Ser 724), and CHOP. (E) Representative electron micrographs are shown for $T s c 2^{-/}, p 53^{-/-}$ MEFs cultured under SO conditions in the presence and absence of $50 \mu \mathrm{M}$ oleic acid. Black arrows indicate the ER. $(F) \mathrm{Tsc}^{-/-}$, $p 53^{-/-}$MEFs were depleted of PERK using siRNA pools and cultured under SO conditions. Viability was assessed by flow cytometry (see also Supplemental Fig. S5E,F). $(G)$ Inhibition of IRE $1 \alpha$ activity rescued the viability of $T s c 2^{-/-}, p 53^{-/-}$MEFs under SO conditions $(P<0.005)$ (see also Supplemental Fig. S6A-D). ( $H)$ Inhibition of IRE1 $\alpha$ activity rescued the viability of $T s c 2^{-/-}, p 53^{-/-}$MEFs under SOG conditions. $\left(^{\star}\right) P<0.005$. promote $T s c 2^{-/-}$, $p 53^{-/-}$cell death. Furthermore, knockdown of the UPR target CHOP, known to induce apoptosis downstream from severely impaired ER function (Ron and Walter 2007), did not consistently restore $T s c 2^{-1-}$ cell viability (Supplemental Fig. S5G,H). As conditions required for IRE1 $\alpha$ pathway activation paralleled the cell death phenotype, we investigated whether the death of $T s c 2^{-/-}$, $p 53^{-/-}$MEFs under SO and SOG limitation was IRE1 $\alpha-$ dependent by using the chemical inhibitor $4 \mu 8 \mathrm{C}$ (Cross et al. 2012). Inhibition of IRE $1 \alpha$ activity, as reflected by 
significantly reduced levels of $\mathrm{XBP}_{\mathrm{s}}$ (Supplemental Fig. $\mathrm{S} 6 \mathrm{~A}, \mathrm{~B})$, increased survival of $T s c 2^{-1-}, p 53^{-/-}$MEFs under SO (Fig. 5G) and SOG conditions (Fig. 5H). Similar results were obtained by depleting IRE1 $\alpha$ expression using multiple lentiviral shRNAs (Supplemental Fig. S6C), which partially restored viability in $\mathrm{Tsc}^{-/-}, \mathrm{p} 53^{-/-} \mathrm{MEFs}$ under SO conditions (Supplemental Fig. S6D). Since JNK is a known mediator of cell death downstream from IRE1 $\alpha$ activation, we tested whether the JNK inhibitor SP600125 could also restore $T s c 2^{-/-}$cell viability (Supplemental Fig. S6E,F). However, we failed to detect any rescue, suggesting that inhibition of JNK activation is not the primary IRE1 $\alpha$ effector controlling $\mathrm{Tsc}^{-/-}, p 53^{-/-}$cell viability under these conditions (Supplemental Fig. S6E,F). In summary, Tsc2-null cells exposed to tumor-like stress undergo an IRE1 $\alpha$ dependent cell death downstream from UPR activation.

Tsc2-deficient tumors exhibit a correlation between markers of hypoxia, mTORC1 signaling, UPR activation, and apoptosis

$\mathrm{TsC2}^{+/-}$mice develop bilateral renal cystic adenomas by 15 mo of age, which is associated with loss of heterozygosity (LOH) of the remaining Tsc2 allele (Onda et al. 1999). Ozcan et al. (2008) have previously demonstrated elevated mTORC1 and UPR activation and apoptosis upon thapsigargin treatment of $\mathrm{Tsc}^{-/-}$tumors. We extended these results in Tsc2-deficient kidney tumors, revealing an in vivo correlation with our in vitro data. To accelerate tumor formation, we treated pregnant $\mathrm{Tsc2}^{+/-}$ mice with the carcinogen N-ethyl-N-nitrosourea (ENU), which promotes LOH (Kobayashi et al. 1999), and examined tumor formation in 3-mo-old offspring. One-hundred percent of treated $T s c 2^{+/-}$mice $(n=20)$ and $0 \%$ of control Tsc ${ }^{+/+}$mice $(n=18)$ displayed kidney tumors (Fig. 6A,B). Serial sections from representative Tsc2-deficient tumors (Fig. 6C) confirmed evidence of apoptosis (TUNEL positivity) (Fig. 6C, black arrow) and constitutive mTORC1 activity (Supplemental Fig. S7A). In addition, qRT-PCR analysis of mRNA isolated from six renal cystic adenomas in 18-mo-old untreated $\mathrm{Tsc}^{+/-}$mice revealed increased expression (1.5-fold to fourfold) of the HIF and/or UPR target genes Pdk1, Ho-1, Xbp $1_{s}, X b p 1_{u,}$ Ero1, and Chop relative to mRNA from age-matched wildtype kidneys (Fig. 6D). In contrast, transcripts encoding CoA:diacylglycerol acyltransferase (DGAT), an enzyme involved in triglyceride synthesis, were expressed at levels corresponding to normal kidney tissue.

In agreement with in vitro experiments using $\mathrm{Tsc}^{-/}$ MEFs, Tsc2 ${ }^{-1-}$ cystic adenomas exhibited distended ER ultrastructure (Fig. 6E), whereas wild-type kidneys displayed normal ER ultrastructure. These in vivo results are consistent with our tissue culture data and demonstrated that markers of HIF, UPR engagement, and cell death are observed in spontaneous $\mathrm{Tsc}^{-1-}$ kidney tumors.

SCD1 inhibition links hypoxic Tsc2 ${ }^{-/-}$cell death and reduced levels of unsaturated lipids under low $\mathrm{O}_{2}$

To confirm that reduced lipid desaturation promotes $\mathrm{TsC}^{-/-}$cell death under SO conditions, we inhibited

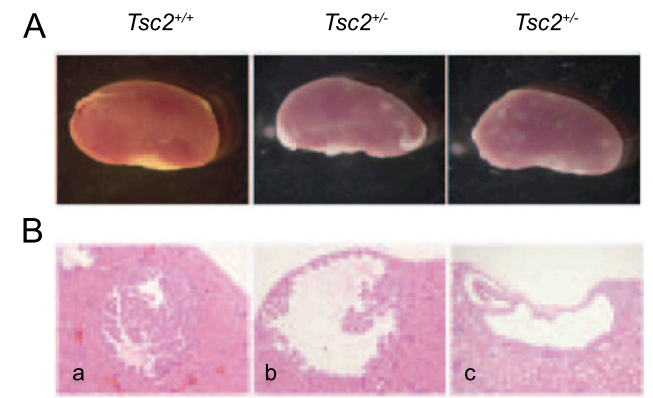

C

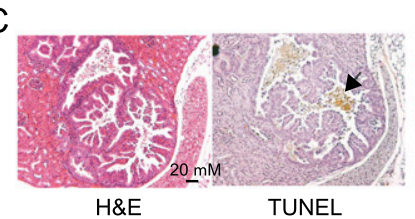

D

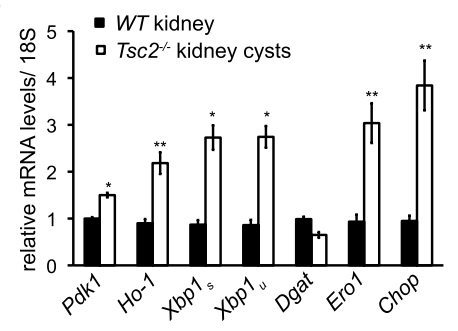

E

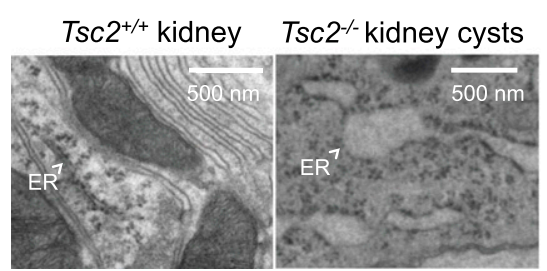

Figure 6. Tsc2-deficient tumors exhibit a correlation between markers of hypoxia, mTORC1 signaling, UPR activation, and apoptosis. (A) Representative kidneys from $\mathrm{Tsc2}^{+/+}$and $\mathrm{Tsc2}^{+/-}$ 3-mo-old mice treated with ENU at embryonic day 14.5 (E14.5). $(B) \mathrm{H} \& \mathrm{E}$ sections of kidney adenomas (panel $a$ ) and cysts (panels $b, c)$ from 3-mo-old $\mathrm{Tsc}^{+/-}$mice treated with ENU at E14.5. (C) Sequential paraffin-embedded tumor sections from a representative murine $\mathrm{Tsc}^{-/-}$kidney adenomatous cyst were assayed for TUNEL positive cells (black arrow). (D) Kidney cysts were dissected from 18-mo-old untreated $\mathrm{Tsc}^{+/-}$mice and kidney tissue from age-matched controls (six tumor and three control samples). RNA was isolated and analyzed for the levels of $P d k 1$, Ho-1, Xbp1 $1_{s}, X b p 1_{u}$, Dgat, Ero1, and CHOP transcripts. $\left(^{\star}\right) P<$ $\left.0.01 ;{ }^{* *}\right) P<0.05$. $(E)$ Representative electron micrographs are shown for $\mathrm{Tsc}^{+/+}$kidney and $T s c 2^{-/-}$kidney cysts. White arrows indicate the ER.

SCD1, the major $\mathrm{O}_{2}$-dependent enzyme catalyzing the rate-limiting reaction of monounsaturated fatty acid synthesis. We employed a pharmacological approach because there are multiple murine SCDs (Scd1-4), making it difficult to effectively reduce SCD1 activity using RNAi techniques. Specifically, we exposed cells to previously described SCD1 inhibitors \{A: CAY10566 from Cayman Chemicals; B: [4-(2-chlorophenoxy)-N-3-(3-methylcarbamoyl) 
phenyl]piperidine-1-carboxamide from BioVision, Inc.; and C: MK-824S from Selleck Bio\}. Normally, Tsc2 ${ }^{-/-}$, $p 53^{-1-}$ MEFs are viable when exposed to limiting serum ("S" conditions) but die under SO limitation; however, Tsc2 ${ }^{-/-}, p 53^{-/-}$MEFs exhibit reduced viability under $\mathrm{S}$ conditions when treated with multiple SCD inhibitors, which can be rescued by the addition of oleic acid (Fig. 7A), demonstrating that reduced lipid desaturation phenocopies $\mathrm{O}_{2}$ limitation under $\mathrm{SO}$ conditions.

Similarly, UPR activation in $\mathrm{Tsc}^{-/-}, \mathrm{p} 53^{-/-}$MEFs cultured under $S$ conditions is magnified when lipid desaturation is reduced by either $\mathrm{O}_{2}$ limitation (Fig. $5 \mathrm{~A}$ ) or treatment with SCD inhibitors (Fig. 7B), an effect that is suppressed by addition of oleic acid. Furthermore, we demonstrated that although normal ER ultrastructure is observed under S conditions, addition of an SCD1 inhibitor produces aberrant ER ultrastructure in $\mathrm{Tsc}^{-/-}$ cells (Fig. 7C) that resembles the ER in Tsc2 $2^{-1-}, p 53^{-/-}$ MEFs cultured under SO conditions (Fig. 2F). These results confirm that unsaturated fatty acids are a critically limiting nutrient for cells with dysregulated growth and demonstrate that hypoxic inhibition of lipid desaturation exacerbates ER stress and promotes apoptosis.

To illuminate the broader pathophysiological importance of this phenotype, we examined whether the survival of multiple human cancer cell lines depends on serum lipids for viability under low $\mathrm{O}_{2}$ (Fig. 7D). Specifically, we showed that human MCF7 breast cancer, RCC10 renal clear cell carcinoma, U251 glioblastoma, HEK293 adenovirus-transformed embryonic kidney cells, and RT4 bladder cancer cells exhibit reduced viability under serum and $\mathrm{O}_{2}$ limitation (Fig. 7D). We assayed the phosphorylation status of downstream mTORC1 effectors in RCC10, U251, and HEK293 tumor cell lines and observed delayed attenuation of 4E-BP1 phosphorylation in RCC10 and U251 but not HEK293 cells (Supplemental Fig. S7B). In agreement with these findings, rapamycin rescued the viability of RCC10 and U251 exposed to SO conditions but not HEK293 cells, demonstrating a mechanistic link between mTORC1 activity and cell death in RCC10 and U251 cells (Fig. 7E). Since mutations in many pathways can drive unregulated growth and proliferation, it is not surprising that we did not see a link between elevated mTORC1 activity and cell death in every cell line. In contrast, cycloheximide rescued the viability of all three cancer cell lines under SO conditions (Fig. 7E). Furthermore all three cells lines tested-RCC10, U251, and HEK292 cells-exhibit evidence of UPR activation (based on $C H O P$ expression) under SO conditions (Supplemental Fig. S7C).

For every human tumor line examined, except MCF7 cells, viability could be rescued by the addition of exogenous lipids (Fig. 7D). Moreover, treatment with SCD1 inhibitor phenocopied $\mathrm{O}_{2}$ deprivation in RCC10, U2S1, and HEK293 cells, which could be rescued by the addition of oleic acid (Fig. 7F). These results demonstrate that cell death under SO limitation is not specific to Tsc2 $2^{-/-}$MEFs and confirm that desaturated lipids are a critically limiting nutrient for hypoxic cell survival in multiple human cancer cell types. We suggest that cells exposed to low $\mathrm{O}_{2}$ rely on lipids in the serum to support growth. Under tumor-like stress, many cancer cells fail to appropriately attenuate growth and proliferation via mutations in mTOR-dependent and -independent pathways and therefore exhibit cell death because they are unable to coordinate protein and lipid synthesis due to a deficiency in desaturated lipids.

\section{Discussion}

A common theme in cancer research is the notion of starving tumor cells by depriving them of an essential nutrient. Most cancer cells are addicted to glucose; they aerobically consume glucose at a surprisingly high rate and secrete glucose-derived carbon as lactate (DeBerardinis et al. 2008). Likewise, oncogenic levels of Myc can render tumor cells critically dependent on not only glucose, but also glutamine, for cell growth, and inhibitors of lactate dehydrogenase A (LDHA) and glutaminase have been shown to have preclinical anti-tumor effects in vivo (Dang 2012). Our results demonstrate that under ischemic stress, desaturated lipids are an essential nutrient for multiple cancer cell types and suggest that targeting enzymes that catalyze lipid desaturation and modify the phospholipid composition of membranes are potential therapeutic targets for cancer treatment.

Deregulation of the mTORC1 pathway drives cell growth and is a common molecular defect in cancer. We began this study with the hypothesis that cells with dysregulated mTORC1 activity would be unable to sustain metabolic growth under various combinations of serum, $\mathrm{O}_{2}$, and glucose limitation. The in vitro stress conditions that we employed mimic the microenvironmental heterogeneity of $\mathrm{O}_{2}$ and nutrient availability in solid tumors, which are characterized by aberrant vascular structure and function (Bertout et al. 2008). Our data indicate that combinations of nutrient stresses, in contrast to the deprivation of a single nutrient, induce unique phenotypes in Tsc2-deficient MEFs; in particular, the combination of either serum and $\mathrm{O}_{2}$ or serum, $\mathrm{O}_{2}$, and glucose limitation induces magnified engagement of the UPR and results in lipid-dependent cell death.

Our data suggest that for cancer cells, which exhibit dysregulated mTORC1, serum lipids are an important source of unsaturated fatty acids under low $\mathrm{O}_{2}$ conditions. In agreement with this, we could restore the viability of $\mathrm{Tsc}^{-/-}, \mathrm{p} 53^{-/-}$MEFs with the addition of unsaturated (at least one double bond) but not saturated (no double bonds) fatty acids. Our NMR data independently highlight the relationship between lipid desaturation and low $\mathrm{O}_{2}$ conditions. When we compared the lipid spectra of de novo synthesized fatty acids from Tsc2-null MEFs exposed to $21 \%$ versus $0.5 \% \mathrm{O}_{2}$, we observed a $50 \%$ decrease in lipid desaturation, which is an $\mathrm{O}_{2}$-dependent process. Furthermore, serum-deprived $\mathrm{Tsc}^{-/-}, \mathrm{p} 53^{-/-}$ MEFs exhibit reduced viability with inhibition of SCD1 or $\mathrm{O}_{2}$ limitation, demonstrating that SCD1 inhibition phenocopies low $\mathrm{O}_{2}$ conditions. In summary, we suggest that dysregulated mTORC1 activity in $T s c 2^{-/-}$MEFs commits cells to a growth program that cannot be 

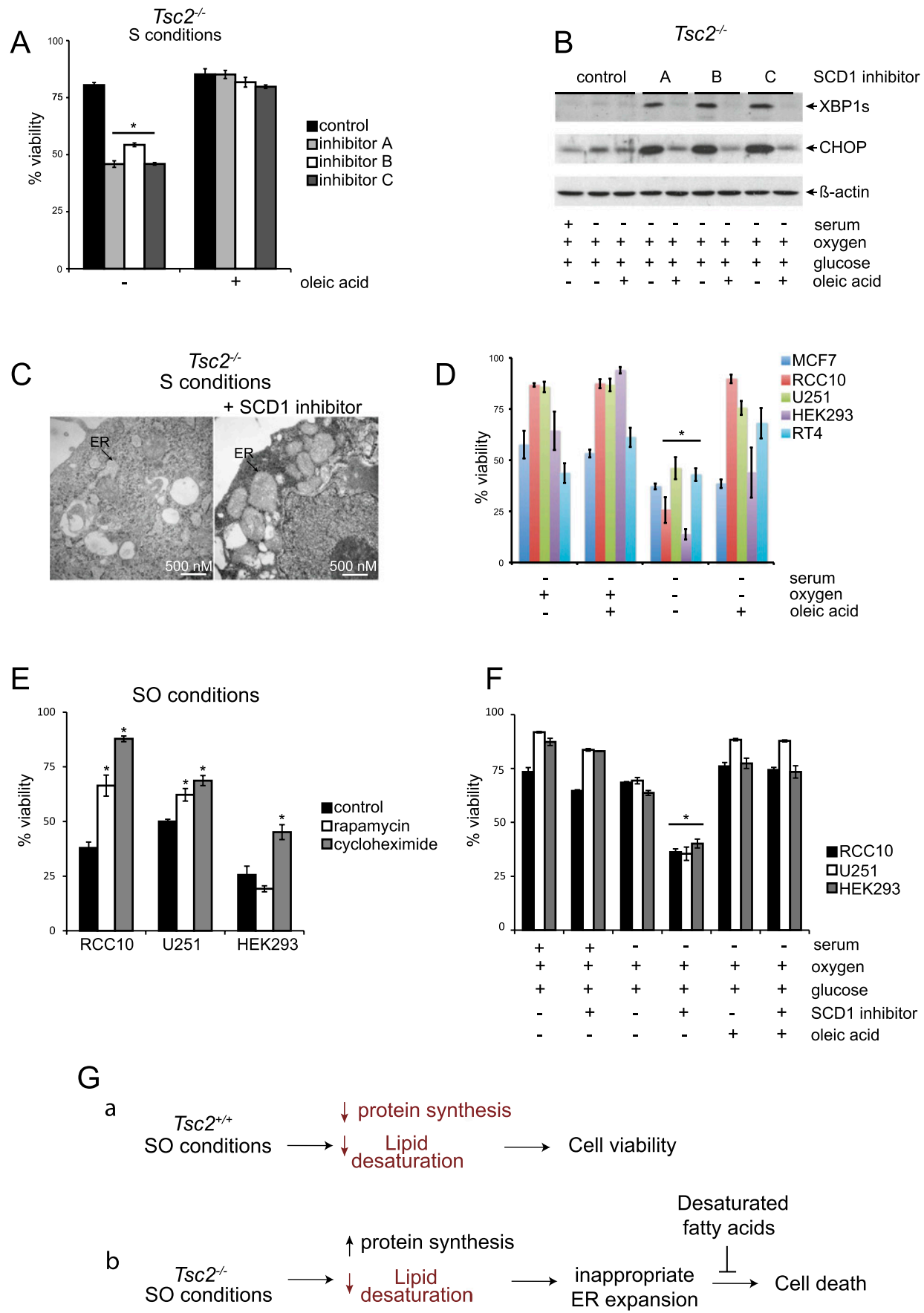

Figure 7. SCD1 inhibition links hypoxic $T s c 2^{-/-}$cell death to reduced levels of unsaturated lipids under low $\mathrm{O}_{2}$. $(A)$ To investigate the link between hypoxic Tsc2 ${ }^{-/-}$cell death and lipid desaturation and test whether oleic acid could reverse cell death, Tsc2 ${ }^{-/-}, p 53^{-/-}$ MEFs were cultured under S conditions with or without three different SCD1 inhibitors (inhibitor A: Cayman Chemical CAY10566, used at $15 \mathrm{nM}$; inhibitor B: BioVision, used at $3 \mu \mathrm{M}$; and inhibitor C: Selleck Chemicals MK-8245, used at $3 \mu \mathrm{M}$ ) and with or without $50 \mu \mathrm{M}$ oleic acid $(P<0.001)$. $(B)$ To determine whether inhibition of SCD1 in Tsc2-null cells alters UPR signaling pathways, Tsc2 ${ }^{-/-}$, $p 53^{-/-}$ MEFs were cultured under S conditions with or without the SCD1 inhibitors A, B, and C described above and oleic acid. Whole-cell extracts were blotted for XBP1 $1_{\mathrm{S}}, \mathrm{CHOP}$, and $\beta$-actin. $(C)$ Representative electron micrographs for Tsc2 ${ }^{-/-}$, p53 ${ }^{-/-}$MEFs cultured under S conditions in the presence and absence of SCD1 inhibitor A are displayed. Black arrows highlight the ER. $(D)$ The viability of human MCF7, RCC10, U251, HEK293, and RT4 cancer cell lines cultured under S or SO conditions in the presence or absence of oleic acid was determined by flow cytometry $(P<0.01)$. (E) The viability of human RCC10, U251, and HEK293 cells after $20 \mathrm{nM}$ rapamycin and $1 \mu M$ cycloheximide treatment was examined after $72 \mathrm{~h}$ of exposure to SO conditions $(P<0.001)$. $(F)$ The viability of human RCC10, U251, and HEK293 cells after treatment with SCD1 inhibitor A (Cayman Chemicals) at $1 \mu \mathrm{M}$ cultured under replete, serum-deprived, and serum- and oxygen-deprived conditions for $72 \mathrm{~h}$ with or without treatment with $50 \mu \mathrm{M}$ oleic acid was examined by flow cytometry $(P<$ 0.001). (G) Model comparing how SO limitation affects stress signaling pathways and viability in $T s c 2^{+/+}, p 53^{-/-}$and Tsc2 $2^{-/-}$, p53 $3^{-/-}$ MEFs. Under SO conditions, levels of desaturated lipids are reduced in $\mathrm{Tsc}^{+/+}, \mathrm{p5} 3^{-/-} \mathrm{MEFs}$; however, this does not affect cell viability

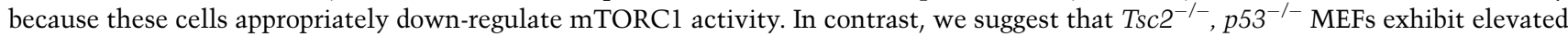
protein synthesis with an increased load of unfolded proteins as well as reduced levels of desaturated lipids under SO conditions. This results in a magnified UPR, which cannot be resolved because the ER is unable to expand appropriately to resolve the increased level of unfolded proteins, leading to loss of cell viability. Addition of unsaturated fatty acids restores the ultrastructure of the ER, dampens the UPR, and rescues cell viability. Pathways or components of the model that are down-regulated are diagrammed in red. 
sustained under conditions of ischemic stress primarily because they cannot generate or maintain sufficient levels of unsaturated fatty acids. While it has been appreciated that fatty acid desaturation is an $\mathrm{O}_{2}$-dependent process, we suggest that under conditions regularly achieved in solid tumors, levels of desaturated lipids become limiting. Importantly, we illustrate the broader pathophysiological importance of this observation by demonstrating that human cancer cells derived from multiple tumor types depend on serum lipids for viability under low $\mathrm{O}_{2}$. Specifically, we show that human RCC10 renal clear cell carcinoma, U251 glioblastoma, HEK293 adenovirus-transformed embryonic kidney, and RT4 bladder cancer cells exhibit reduced viability under SO conditions, which can be rescued by the addition of exogenous desaturated lipids.

mTORC1 promotes cell growth through the modulation of protein synthesis, and unregulated protein synthesis in Tsc2-null cells has been linked to activation of the UPR (Ozcan et al. 2008; Kang et al. 2011). In addition, dysregulated mTORC1 activates SREBP-dependent de novo lipid biosynthesis, and silencing of SREBP attenuates mTORC1-mediated increases in cell size (Porstmann et al. 2008; Duvel et al. 2010; Griffiths et al. 2013). These results suggest that $\mathrm{mTORC} 1$ regulates both protein and lipid synthesis and that both pathways are necessary for cell growth. In this study, we speculate that $T s c 2^{-/-}$, $p 53^{-1-}$ MEFs die under tumor-like stress because they exhibit increased protein synthesis without a concomitant increase in lipid synthesis. In other words, reduced levels of desaturated lipids impair the ability of $T s c 2^{-1-}$, $p 53^{-/-}$MEFs to cope with an increased load of unfolded proteins, and this disconnect between protein and lipid synthesis under low $\mathrm{O}_{2}$ results in Tsc2-null cell death. It is important to note that both protein synthesis and lipid desaturation occur on the ER membrane. Under serum and $\mathrm{O}_{2}$ limitation, $\mathrm{Tsc}^{+/+}$, $p 53^{-/-}$cells displayed reduced levels of desaturated lipids; however, this does not lead to cell death because these cells appropriately downregulate mTORC1 (Fig. 7G). In contrast, attenuation of mTORC1 activity is delayed under serum and $\mathrm{O}_{2}$ limitation in $\mathrm{Tsc}^{-/-}, \mathrm{p} 53^{-/-}$MEFs, which results in an increase in the load of unfolded proteins and magnification of the UPR (Fig. 7G). However, we suggest that insufficient levels of desaturated lipids disrupt appropriate ER expansion and resolution of the UPR, resulting in IRE $1 \alpha-$ dependent cell death. In support of this notion, UPR activation, ER expansion, and $T s c 2^{-/-}$cell death can be rescued by the addition of unsaturated fatty acids. Several recent reports have demonstrated that hypoxic cells require glutamine to maintain de novo lipid synthesis, as hypoxia limits de novo lipid synthesis from glucose (Metallo et al. 2011; Wise et al. 2011). In this study, we reveal that hypoxia also regulates lipid synthesis downstream from glucose and glutamine uptake. Specifically, we demonstrate that the desaturation of newly synthesized lipids is inhibited under hypoxic conditions, which results in altered ER expansion and a maladaptive UPR in Tsc2 $2^{-/-}$cells, making them critically dependent on exogenous desaturated fatty acids for survival.
The relationship between the synthesis, storage, and metabolism of free fatty acids in tumor cells is unclear (Yecies and Manning 2010). Most aggressive cancers exhibit increased levels of de novo fatty acid synthesis (Kuhajda 2000), which could be due to SREBP activation in tumors with dysregulated mTORC1 (Duvel et al. 2010). Furthermore, pharmacological inhibition of de novo fatty acid synthesis can preferentially kill glycolytic cancer cell lines and limit the growth of xenograft tumors (Hatzivassiliou et al. 2005; Menendez and Lupu 2007). In addition, many highly aggressive human cancers exhibit an increase in the release of fatty acids from lipid stores. Monoacylglycerol lipase (MAGL) regulates the release of fatty acids from neutral and phospholipid stores and is highly expressed in aggressive human cancers (Nomura et al. 2010). A high-fat diet can contribute to malignancy in cancers lacking MAGL, revealing a role for exogenous sources of fatty acids in cancer pathology (Nomura et al. 2010). At present, it is unclear whether newly synthesized fatty acids, serum fatty acids, and/or fatty acids released from neutral and phospho-stores all enter the same interchangeable cellular pool or whether there are distinctions between fatty acid sources that are critical for lipid metabolism or signaling in cancer cells (Yecies and Manning 2010).

In conclusion, our data underscore the importance of unsaturated serum lipids to the survival of hypoxic cancer cell lines and suggest that unsaturated fatty acids can be limiting in the microenvironment of hypoxic tumors. These results support the notion that targeting lipid metabolism within the tumor microenvironment could be an important cytotoxic therapy for tumors with dysregulated mTORC1, as opposed to using rapamycin, which provides largely cytostatic benefits to cancer patients.

\section{Materials and methods}

\section{Cell culture and cell viability assays}

Tsc2 $2^{+/+}, p 53^{-/-}$and Tsc2 $2^{-/-}, p 53^{-/-}$MEFs were kindly provided by David Kwiatkowski (Harvard Medical School), and the Perk ${ }^{+/+}$ and Perk ${ }^{-1}$ MEFs were obtained from the Diehl laboratory (Bobrovnikova-Marjon et al. 2010). For colony-forming assays, cells were exposed to replete medium (DMEM 10\% FBS, $5 \mathrm{mM}$ glucose), S (DMEM 0.5\% FBS, $5 \mathrm{mM}$ glucose) and SG (DMEM $0.5 \%$ FBS, $0.5 \mathrm{mM}$ glucose) conditions at $21 \%, 3 \%, 1.5 \%$, and $0.5 \% \mathrm{O}_{2}$ for $48 \mathrm{~h}$ and then allowed to grow under replete conditions for seven additional days at $21 \% \mathrm{O}_{2}$. Colonies were stained with $4 \%$ crystal violet. For survival experiments, $\mathrm{Tsc}^{+/+}$, $p 53^{-/-}$and Tsc2 $2^{-/-}, p 53^{-/-}$MEFs were exposed to replete medium (DMEM 10\% FBS, $25 \mathrm{mM}$ glucose), S (DMEM 0.5\% FBS, $25 \mathrm{mM}$ glucose), and SG (DMEM 0.5\% FBS, $0.5 \mathrm{mM}$ glucose) conditions at $21 \%$ and $0.5 \% \mathrm{O}_{2}$ for $48 \mathrm{~h}$, and viability was determined by the percentage of cells that were double negative for Annexin V-FITC and propidium iodide (PI) staining (BD Biosciences).

To prepare RNA or whole-cell extracts, cells were plated the night before at $0.5 \times 10^{6}$ cells per $10-\mathrm{cm}^{2}$ plate and then exposed to replete medium, S (DMEM 0.5\% FBS, $25 \mathrm{mM} \mathrm{FBS),} \mathrm{or} \mathrm{SG}$ (DMEM 0.5\% FBS, $0.5 \mathrm{mM}$ glucose) conditions between 0 and $30 \mathrm{~h}$. Cells were maintained at an exponential growth phase for viability assays and RNA and protein isolation. Low $\mathrm{O}_{2}$ condi- 
tions $(0.5 \%)$ were achieved in a Ruskinn in vivO ${ }_{2} 400$ workstation by supplementing ambient air with $\mathrm{N}_{2}$ and $\mathrm{CO}_{2}$. To compare normoxic and hypoxic conditions, monolayers were never allowed to exceed $70 \%$ confluence. To achieve $3 \%$ and $1.5 \% \mathrm{O}_{2}$, cells were cultured in Hereaus incubators (Thermo Scientific).

Lipids

Lipid-reduced FBS was purchased from Thermo Scientific HyClone, and BSA-conjugated oleic/ linoleic acid was purchased from Sigma. To separately conjugate palmitate or oleate to BSA, a stock solution of $72 \mu \mathrm{L}$ of $250 \mathrm{mM}$ palmitate in $\mathrm{EtOH}$ or 57.12 $\mu \mathrm{L}$ of $315 \mathrm{mM}$ oleate in $\mathrm{EtOH}$ was mixed with $4 \mathrm{~mL}$ of $10 \%$ BSA (fraction $\mathrm{V}$ ) for $1 \mathrm{~h}$ at $37^{\circ} \mathrm{C}$ and stored at $4^{\circ} \mathrm{C}$.

\section{Glucose uptake}

Tsc2 $2^{-/-}, p 53^{-/-}$MEFs were plated at $0.5 \times 10^{6}$ cells per six-well plate. The following day, cells in triplicate wells were exposed to $21 \%$ and $0.5 \% \mathrm{O}_{2}$ in DMEM $5 \mathrm{mM}$ glucose and $10 \%$ or $0.5 \%$ FBS. After $16 \mathrm{~h}$, glucose uptake was measured using the Nova Biomedical Flex Analyzer and expressed as millimoles consumed per $10^{5}$ cells.

\section{Electron microscopy}

Samples for electron microscopy were fixed in $2.5 \%$ glutaraldehyde and $2.0 \%$ paraformaldehyde in $0.1 \mathrm{M}$ sodium cacodylate buffer ( $\mathrm{pH} 7.4)$ overnight at $4^{\circ} \mathrm{C}$ and processed as described previously (Buzzai et al. 2007).

\section{ATP measurements}

Levels of ATP in Tsc2 $2^{+/}, p 53^{-/-}$and $T s c 2^{-/-}, p 53^{-/-}$MEFs were determined by an ATP bioluminescence assay kit CLS II (Roche Applied Sciences) and normalized for cell number. The boiling method described in the kit protocol was used to lyse the cells.

\section{$q R T-P C R$}

Total RNA was isolated from cells using the Trizol reagent protocol (Invitrogen) and from kidney tissue using an RNAeasy minikit and the supplier's protocol (Qiagen). cDNA was synthesized from a high-capacity RNA-to-cDNA kit from Applied Biosystems. qRT-PCR analysis was performed in an Applied Biosystems $7900 \mathrm{HT}$ sequence detection system and normalized to $18 \mathrm{~S}$ RNA.

\section{$N M R$}

Relative lipid synthesis rates were determined in $225-\mathrm{cm}^{2}$ T-flasks (T-225). Cultures were plated at an initial density of $2 \times 10^{6}$ to $3 \times 10^{6}$ cells. After $\sim 16$ h of growth in DMEM with $10 \%$ FBS, the cultures were washed with DMEM containing no glucose, glutamine, or serum. Subsequently, they were incubated with $35 \mathrm{~mL}$ of DMEM containing $10 \mathrm{mM}$ glucose, $3 \mathrm{mM}$ glutamine, and 10\% dialyzed FBS (Gemini) for $24 \mathrm{~h}$. Flasks contained either $\left[\mathrm{U}^{-13} \mathrm{C}_{6}\right]$ glucose and unenriched glutamine or unenriched glucose and $\left[\mathrm{U}_{-}{ }^{13} \mathrm{C}_{5}\right.$ ]glutamine (Isotec). At the end of the 24-h labeling period, cultures were washed twice with PBS. The first PBS wash included $1 \%$ fatty acid-free albumin to adsorb any extracellular fats, and the second contained no albumin. The cells were trypsinized and suspended in $10 \mathrm{~mL}$ of $1 \%$ fatty acidfree albumin in PBS. The final cell number was determined with a hemocytometer, and the cells were recovered by centrifugation and frozen at $-80^{\circ} \mathrm{C}$.
Total cellular lipids, both polar and nonpolar, were extracted with the Bligh-Dyer procedure (Bligh and Dyer 1959). NMR spectra were acquired with a 9.4 Tesla Varian DirectDrive spectrometer and a 5-mm high-resolution probe (Varian, Inc.). For ${ }^{13} \mathrm{C}$ spectra, bilevel WALTZ16 ${ }^{1} \mathrm{H}$ decoupling was used to eliminate ${ }^{1} \mathrm{H}_{-}{ }^{13} \mathrm{C}$ couplings and enhance the signal to noise ratio by the Nuclear Overhauser Effect. The acquisition parameters were as follows: $60^{\circ}$ pulse angle, 3.1 -sec relaxation delay, $30,000-\mathrm{Hz}$ spectral width, and 14,000 transients.

\section{FAME analysis}

GC-MS analysis was used to examine total cellular fatty acids. Following extraction, lipids were converted to fatty acid methyl esters so that they could be separated by gas chromatography. Tsc2 ${ }^{-1-}, p 53^{-/-}$MEFs were cultured under S or SO conditions with or without oleic acid in medium that contained $\left[1,6{ }^{13} \mathrm{C}_{2}\right]$ glucose and then extracted with a standard chloroform/methanol procedure (Bligh and Dyer 1959). The chloroform fraction was dried under a stream of nitrogen and then redissolved in a 4:1 mixture of methanol and toluene. Acetylchloride (14 mM) was added to produce catalytic $\mathrm{H}^{+}$in situ, and butylated hydroxytoluene $(0.45 \mathrm{mM})$ was added to protect unsaturated fats from oxidation. The mixture was heated for $2 \mathrm{~h}$ at $100^{\circ} \mathrm{C}$ in a sealed glass tube. After cooling, the solution was mixed with $0.56 \mathrm{M}$ aqueous sodium carbonate at a ratio of 2:5 to produce hydrophobic droplets that were rich in toluene and the fatty acid methyl esters. Centrifugation at $10,000 \mathrm{~g}$ resulted in droplet coalescence. Two microliters of the hydrophobic phase was injected (splitless mode) into an Agilent 5975C GC-MS system equipped with a single quadrupole mass detector. Electron impact was used to produce charged species. A polar DB-5 column was used for chromatography. Mass spectra were quantified with the MSD ChemStation software from Agilent. Isocor, written for the Python programming environment (http://www.python.org), was used to correct mass spectra for natural abundance contributions from ${ }^{13} \mathrm{C}$.

\section{Statistical analysis}

Error bars represent standard error of the mean for Figures 1, B and $\mathrm{C} ; 2 \mathrm{~F} ; 6 \mathrm{D}$; and $7 \mathrm{~B}$ and standard deviation for the remaining graphs. Statistical analyses were performed by two two-tailed Student's $t$-tests.

\section{Acknowledgments}

We thank members of the Simon, Thompson, and Birnbaum laboratories for helpful discussions; Chad B. Stein for technical assistance; and Almut Schulze for sharing unpublished data. We are grateful to Qian-Chun Yu and the Electron Microscopy and Histology cores for their assistance; C.B. Thompson, D. Kwiatkowski, D. Sabatini, and B. Manning for providing reagents; and D. Ron for sharing his IRE1 inhibitor, $4 \mu 8 \mathrm{C}$, before publication. This work was funded in whole or in part by National Institutes of Health Grants P01 CA104838 (National Cancer Institute [NCI], to M.C.S.) and CA1048387-03S1 (NCI, to R.M.Y.). The Howard Hughes Medical Institute and the Abramson Family Cancer Research Institute also supported this work. M.C.S. is an investigator of the Howard Hughes Medical Institute.

\section{References}

Bertout JA, Patel SA, Simon MC. 2008. The impact of $\mathrm{O}_{2}$ availability on human cancer. Nat Rev Cancer 8: 967-975. 
Bligh EG, Dyer WJ. 1959. A rapid method of total lipid extraction and purification. Can I Biochem Physiol 37: 911-917.

Bobrovnikova-Marion E, Grigoriadou C, Pytel D, Zhang F, Ye J, Koumenis C, Cavener D, Diehl JA. 2010. PERK promotes cancer cell proliferation and tumor growth by limiting oxidative DNA damage. Oncogene 29: 3881-3895.

Brugarolas J, Lei K, Hurley RL, Manning BD, Reiling JH, Hafen E, Witters LA, Ellisen LW, Kaelin WG Jr. 2004. Regulation of mTOR function in response to hypoxia by REDD1 and the TSC1/TSC2 tumor suppressor complex. Genes Dev 18: 2893-2904.

Buzzai M, Jones RG, Amaravadi RK, Lum JJ, DeBerardinis RJ, Zhao F, Viollet B, Thompson CB. 2007. Systemic treatment with the antidiabetic drug metformin selectively impairs p53deficient tumor cell growth. Cancer Res 67: 6745-6752.

Choo AY, Kim SG, Vander Heiden MG, Mahoney SJ, Vu H, Yoon SO, Cantley LC, Blenis J. 2010. Glucose addiction of TSC null cells is caused by failed mTORC1-dependent balancing of metabolic demand with supply. Mol Cell 38: 487-499.

Crino PB, Nathanson KL, Henske EP. 2006. The tuberous sclerosis complex. N Engl J Med 355: 1345-1356.

Cross BC, Bond PJ, Sadowski PG, Jha BK, Zak J, Goodman JM, Silverman RH, Neubert TA, Baxendale IR, Ron D, et al. 2012. The molecular basis for selective inhibition of unconventional mRNA splicing by an IRE1-binding small molecule. Proc Natl Acad Sci 109: E869-E878.

Dang CV. 2012. Links between metabolism and cancer. Genes Dev 26: 877-890.

DeBerardinis RJ, Lum JJ, Hatzivassiliou G, Thompson CB. 2008. The biology of cancer: Metabolic reprogramming fuels cell growth and proliferation. Cell Metab 7: 11-20.

DeYoung MP, Horak P, Sofer A, Sgroi D, Ellisen LW. 2008. Hypoxia regulates TSC1/2-mTOR signaling and tumor suppression through REDD1-mediated 14-3-3 shuttling. Genes Dev 22: 239-251.

Diakogiannaki E, Welters HJ, Morgan NG. 2008. Differential regulation of the endoplasmic reticulum stress response in pancreatic $\beta$-cells exposed to long-chain saturated and monounsaturated fatty acids. I Endocrinol 197: 553-563.

Duvel K, Yecies JL, Menon S, Raman P, Lipovsky AI, Souza AL, Triantafellow E, Ma Q, Gorski R, Cleaver S, et al. 2010. Activation of a metabolic gene regulatory network downstream of mTOR complex 1. Mol Cell 39: 171-183.

Egan DF, Shackelford DB, Mihaylova MM, Gelino S, Kohnz RA, Mair W, Vasquez DS, Joshi A, Gwinn DM, Taylor R, et al. 2011. Phosphorylation of ULK1 (hATG1) by AMP-activated protein kinase connects energy sensing to mitophagy. Science 331: 456-461.

Griffiths B, Lewis C, Bensaad K, Ros S, Zhang Q, Ferber EC, Konisti S, Peck B, Miess H, East P, et al. 2013. Sterol regulatory element binding protein-dependent regulation of lipid synthesis supports cell survival and tumor growth. Cancer Metab 1: 3.

Guertin DA, Sabatini DM. 2007. Defining the role of mTOR in cancer. Cancer Cell 12: 9-22.

Gwinn DM, Shackelford DB, Egan DF, Mihaylova MM, Mery A, Vasquez DS, Turk BE, Shaw RJ. 2008. AMPK phosphorylation of raptor mediates a metabolic checkpoint. Mol Cell 30: 214-226.

Hatzivassiliou G, Zhao F, Bauer DE, Andreadis C, Shaw AN, Dhanak D, Hingorani SR, Tuveson DA, Thompson CB. 2005. ATP citrate lyase inhibition can suppress tumor cell growth. Cancer Cell 8: 311-321.

Inoki K, Zhu T, Guan KL. 2003. TSC2 mediates cellular energy response to control cell growth and survival. Cell 115: 577-590.
Kang YJ, Lu MK, Guan KL. 2011. The TSC1 and TSC2 tumor suppressors are required for proper ER stress response and protect cells from ER stress-induced apoptosis. Cell Death Differ 18: 133-144.

Kim J, Guan KL. 2011. Regulation of the autophagy initiating kinase ULK1 by nutrients: Roles of mTORC1 and AMPK. Cell Cycle 10: 1337-1338.

Kobayashi T, Minowa O, Kuno J, Mitani H, Hino O, Noda T. 1999. Renal carcinogenesis, hepatic hemangiomatosis, and embryonic lethality caused by a germ-line Tsc2 mutation in mice. Cancer Res 59: 1206-1211.

Kuhajda FP. 2000. Fatty-acid synthase and human cancer: New perspectives on its role in tumor biology. Nutrition 16: 202208.

Laplante M, Sabatini DM. 2012. mTOR signaling in growth control and disease. Cell 149: 274-293.

Liu L, Cash TP, Jones RG, Keith B, Thompson CB, Simon MC. 2006. Hypoxia-induced energy stress regulates mRNA translation and cell growth. Mol Cell 21: 521-531.

Ma XM, Blenis J. 2009. Molecular mechanisms of mTORmediated translational control. Nat Rev Mol Cell Biol 10: 307-318.

Manning BD, Tee AR, Logsdon MN, Blenis J, Cantley LC. 2002. Identification of the tuberous sclerosis complex-2 tumor suppressor gene product tuberin as a target of the phosphoinositide 3-kinase/akt pathway. Mol Cell 10: 151-162.

Menendez JA, Lupu R. 2007. Fatty acid synthase and the lipogenic phenotype in cancer pathogenesis. Nat Rev Cancer 7: 763-777.

Menon S, Manning BD. 2008. Common corruption of the mTOR signaling network in human tumors. Oncogene 27: S43-S51.

Metallo CM, Gameiro PA, Bell EL, Mattaini KR, Yang J, Hiller K, Jewell CM, Johnson ZR, Irvine DJ, Guarente L, et al. 2011. Reductive glutamine metabolism by IDH1 mediates lipogenesis under hypoxia. Nature 481: 380-384.

Nomura DK, Long JZ, Niessen S, Hoover HS, Ng SW, Cravatt BF. 2010. Monoacylglycerol lipase regulates a fatty acid network that promotes cancer pathogenesis. Cell 140: 49-61.

Onda H, Lueck A, Marks PW, Warren HB, Kwiatkowski DJ. 1999. Tsc $2^{+/-}$mice develop tumors in multiple sites that express gelsolin and are influenced by genetic background. J Clin Invest 104: 687-695.

Ozcan U, Ozcan L, Yilmaz E, Duvel K, Sahin M, Manning BD, Hotamisligil GS. 2008. Loss of the tuberous sclerosis complex tumor suppressors triggers the unfolded protein response to regulate insulin signaling and apoptosis. Mol Cell 29: $541-551$.

Porstmann T, Santos CR, Griffiths B, Cully M, Wu M, Leevers S, Griffiths JR, Chung YL, Schulze A. 2008. SREBP activity is regulated by $\mathrm{mTORC} 1$ and contributes to Akt-dependent cell growth. Cell Metab 8: 224-236.

Ron D, Walter P. 2007. Signal integration in the endoplasmic reticulum unfolded protein response. Nat Rev Mol Cell Biol 8: 519-529.

Seagroves TN, Ryan HE, Lu H, Wouters BG, Knapp M, Thibault P, Laderoute K, Johnson RS. 2001. Transcription factor HIF-1 is a necessary mediator of the pasteur effect in mammalian cells. Mol Cell Biol 21: 3436-3444.

Sheen JH, Zoncu R, Kim D, Sabatini DM. 2011. Defective regulation of autophagy upon leucine deprivation reveals a targetable liability of human melanoma cells in vitro and in vivo. Cancer Cell 19: 613-628.

Thoreen CC, Kang SA, Chang JW, Liu Q, Zhang J, Gao Y, Reichling LJ, Sim T, Sabatini DM, Gray NS. 2009. An ATPcompetitive mammalian target of rapamycin inhibitor reveals 
rapamycin-resistant functions of mTORC1. I Biol Chem 284: 8023-8032.

Vousden KH, Prives C. 2009. Blinded by the light: The growing complexity of p53. Cell 137: 413-431.

Wise DR, Ward PS, Shay JE, Cross JR, Gruber JJ, Sachdeva UM, Platt JM, DeMatteo RG, Simon MC, Thompson CB. 2011. Hypoxia promotes isocitrate dehydrogenase-dependent carboxylation of $\alpha$-ketoglutarate to citrate to support cell growth and viability. Proc Natl Acad Sci 108: 19611-19616.

Wouters BG, Koritzinsky M. 2008. Hypoxia signalling through $\mathrm{mTOR}$ and the unfolded protein response in cancer. Nat ReV Cancer 8: 851-864.

Wullschleger S, Loewith R, Hall MN. 2006. TOR signaling in growth and metabolism. Cell 124: 471-484.

Yecies JL, Manning BD. 2010. Chewing the fat on tumor cell metabolism. Cell 140: 28-30.

Yip CK, Murata K, Walz T, Sabatini DM, Kang SA. 2010. Structure of the human mTOR complex I and its implications for rapamycin inhibition. Mol Cell 38: 768-774.

Zhang H, Cicchetti G, Onda H, Koon HB, Asrican K, Bajraszewski N, Vazquez F, Carpenter CL, Kwiatkowski DJ. 2003. Loss of Tsc1/Tsc2 activates mTOR and disrupts PI3K-Akt signaling through downregulation of PDGFR. I Clin Invest 112: 12231233. 


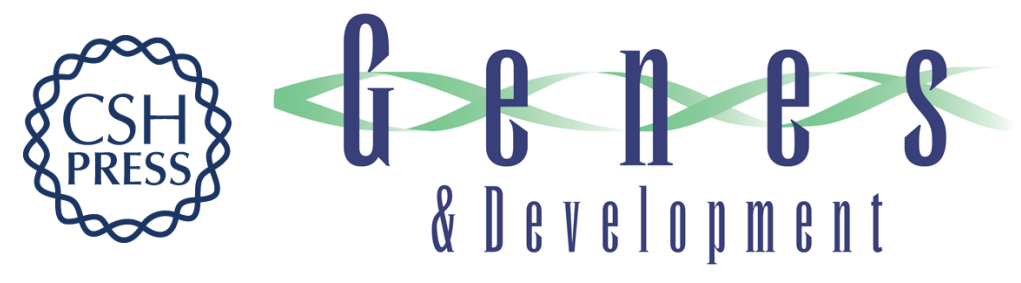

\section{Dysregulated $\mathrm{mTORC1}$ renders cells critically dependent on desaturated lipids for survival under tumor-like stress}

Regina M. Young, Daniel Ackerman, Zachary L. Quinn, et al.

Genes Dev. 2013, 27:

Access the most recent version at doi:10.1101/gad.198630.112

Supplemental http://genesdev.cshlp.org/content/suppl/2013/05/22/27.10.1115.DC1
Material

References This article cites 45 articles, 11 of which can be accessed free at:

http://genesdev.cshlp.org/content/27/10/1115.full.html\#ref-list-1

License

Email Alerting Receive free email alerts when new articles cite this article - sign up in the box at the top

Service

right corner of the article or click here.

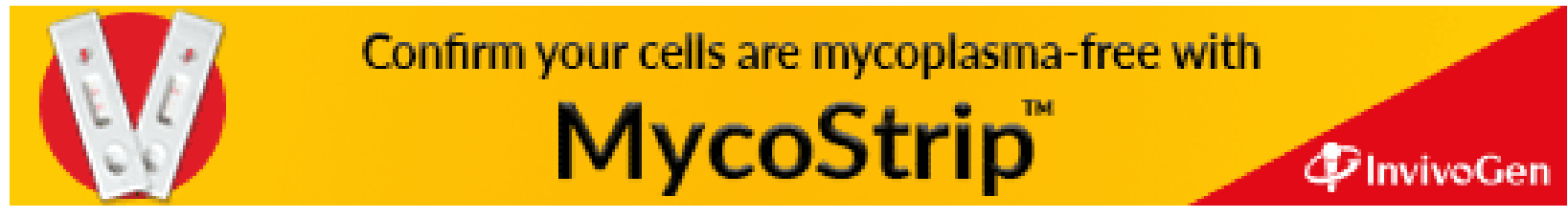

